\title{
Complex Scaling Approach for Stability Analysis and Stabilization of Multiple Time-Delayed Systems
}

\author{
Jun Zhou $(\mathbb{D}$, Ketian Gao $\mathbb{D}$, and Xinbiao Lu $(\mathbb{D}$ \\ Department of Automatic Control Engineering, School of Energy and Electrical Engineering, Hohai University, Nanjing 211100, China \\ Correspondence should be addressed to Jun Zhou; katsura@hhu.edu.cn
}

Received 7 January 2018; Revised 12 February 2018; Accepted 27 February 2018; Published 12 April 2018

Academic Editor: Filippo Cacace

Copyright (c) 2018 Jun Zhou et al. This is an open access article distributed under the Creative Commons Attribution License, which permits unrestricted use, distribution, and reproduction in any medium, provided the original work is properly cited.

\begin{abstract}
Based on complex scaling, the paper copes with stability analysis and stabilization of linear time-invariant continuous-time systems with multiple time delays in state, control input, and measured output, under state and/or output feedback. More specifically, the paper establishes stability criteria for exponential/asymptotical stability with Hurwitz complex scaling being applied to the related characteristic polynomials. The stability conditions are necessary and sufficient, delay-dependent, and independent of feedback structures and open-loop poles distribution. The criteria can be implemented graphically with locus plotting or numerically without it; moreover, no prior frequency sweeping is involved, and the contour and locus encirclement orientations can be self-defined. Exploiting the complex scaling approach and embracing its technical merits, it is considered to design static state feedback control for robustly stabilizing time-delayed systems. A small-gain interpretation for the suggested stabilization is also elaborated. Examples are included to illustrate the main results.
\end{abstract}

\section{Introduction}

This study is motivated by the fact that many frequencydomain stability criteria are developed and exploited as parametric and graphical tools for stability analysis and stabilization in classes of time-delayed systems, subject to various structural, analytical or technical assumptions, and constraints. Due to intuitive conception, simple geometry, experimental availability, and frequency-domain methods are extended to stability analysis and stabilization in distributed $[1,2]$, periodically time-varying [3], and timedelayed ones with or without uncertainties [4-9]. Some stability criteria are given in Nyquist-like form, which involve open-loop unstable poles and frequency-domain plots in general and thus are not useful for time-delayed systems with time-varying factors [10-12]. However, when time-delayed systems are dealt with [13-17], poles distribution is not available numerically and frequency responses need to be examined with prior sweeping. Stability criteria for timedelayed systems are created also via LMI $[18,19]$. Complexvalued neural networks with time delays are attached in [2022]. As a matter of fact, when time delay occurs in dynamics and/or their derivatives, the characteristic polynomials are quasi-polynomials in one variable and its exponential powers $[4,23]$ that are of infinite degree. Although finitely many poles can be computed by truncating infinite series of exponentials or other numerical algorithms, approximate poles are not meaningful for analytically exploiting Nyquist-type criteria, let alone unavoidable algorithm convergence [24] and numerical pathology.

Lately, a series of studies [25-28] are developed in various systems based on complex scaling, which enables us to cope with structure and stability issues by means of frequencydomain properties of the concerned systems without invoking pole distribution and specific frequency responses. More precisely, the study [25] talks about stability of sampleddata systems; the work [26] deals with that of continuoustime fractal-order systems; the study [27] considers the Lur'etype feedback nonlinear systems. The paper [28] generalizes the complex scaling tool for investigating structural features in LTI systems, say controllability, observability, and so on. Tentative discussion via complex scaling for stability in openloop time-delayed systems was reported in [29]. In this study, we carefully work through the technical details and provide rigorous proofs for internal stability criteria for LTI feedback systems with multiple time delays. 
More specifically, to surmount multiple time-delay dependence, infinite-dimensionality, polynomial cancellation, and other complex analysis issues in quasi characteristic polynomials of time-delayed continuous systems in feedback configuration, the complex scaling technique is coined by using auxiliary Hurwitz polynomial to modify the return difference relationships such that the modified ones reflect exactly and completely asymptotical/exponential stability while analytical properties are retained for applying the argument principle [30]. The suggested stability criteria produce necessary and sufficient conditions for asymptotical and exponential stability (or simply internal stability), which involve neither open-loop unstable poles that are hard to know, nor prespecified contour and locus encirclement orientations. The stability criteria are implementable graphically with locus plotting or merely by numerical complex argument integration. Also using the complex scaling approach, we further contrive a new technique for stabilizing timedelayed systems with static state feedback. The stabilization results in asymptotical/exponential stability internally as well as $L_{2}$-stability externally that are robust with respect to time delay.

Outlines. Section 2 collects preliminaries to LTI time-delayed continuous systems of retarded type, their feedback configuration, and return difference relationships. Section 3 states the complex scaling return difference relationships and proves the generalized criteria. Stabilization is explained in Section 4. Illustrations are sketched in Section 5, and Section 6 gives our conclusions.

Notations 1. $\mathscr{R}$ and $\mathscr{C}$ are the sets of real and complex numbers, respectively, while $\mathscr{C}^{+}=\{s \in \mathscr{C}: \operatorname{Re}(s)>0\} . I_{i}$ is the $i \times i$ identity matrix. $(\cdot)^{*}$ means the complex conjugate transpose of a matrix $(\cdot) \cdot \operatorname{det}(\cdot)$ means the determinant of a square matrix $(\cdot)$. A polynomial is Hurwitz polynomial if all its roots have negative real parts, and $\operatorname{deg}(\cdot)$ means its degree. $L_{2}[0, \infty)$ denotes the functions space such that $f(\cdot) \in$ $L_{2}[0, \infty)$ means that $\|f(\cdot)\|_{L_{2}[0, \infty)}^{2}=\int_{0}^{\infty}\|f(\tau)\|^{2} d \tau<\infty$, where $\|\cdot\|$ is the Euclidean norm of a vector $(\cdot)$ or the induced matrix norm as appropriately.

\section{Preliminaries to Time-Delayed Systems}

2.1. Linear Time-Delayed Continuous-Time Systems of Retarded Types. By linear time-delayed continuous-time system of retarded type, we mean a system in form of

$$
\begin{aligned}
& \Sigma_{G}: \\
& \left\{\begin{array}{l}
\dot{x}(t)=A_{0} x(t)+\sum_{i=1}^{\kappa} A_{i} x\left(t-\tau_{i}\right)+B_{0} u(t)+\sum_{i=1}^{\kappa} B_{i} u\left(t-\sigma_{i}\right) \\
y(t)=C_{0} x(t)+\sum_{i=1}^{\kappa} C_{i} x\left(t-v_{i}\right)+D u(t)
\end{array}\right.
\end{aligned}
$$

where $x(t) \in \mathscr{R}^{n}, u(t) \in \mathscr{R}^{m}$, and $y(t) \in \mathscr{R}^{l}$ are the state, control input, and measured output, respectively. $\kappa>0$ is an integer, and $A_{i} \in \mathscr{R}^{n \times n}, B_{i} \in \mathscr{R}^{n \times m}, C_{i} \in \mathscr{R}^{l \times n}$, and
$D \in \mathscr{R}^{l \times m}$, respectively, are constant matrices. In (1), the time delays are assumed to satisfy

$$
\begin{aligned}
& \tau: 0<\tau_{1}<\tau_{2}<\cdots<\tau_{\kappa}, \\
& \sigma: 0<\sigma_{1}<\sigma_{2}<\cdots<\sigma_{\kappa} \\
& \nu: 0<\nu_{1}<\nu_{2}<\cdots<\nu_{\kappa}, \\
& \tau_{\min }=\tau_{1}>0, \\
& \tau_{\max }=\tau_{\kappa}>0 .
\end{aligned}
$$

That is, $\tau, \sigma$, and $\nu$ are short notations for the corresponding multiple time delays.

The transfer function matrix of system (1) is

$$
\begin{aligned}
G(s, \tau, \sigma, \nu) & =C(s, \nu) \Delta_{G}^{-1}(s, \tau) B(s, \sigma)+D, \\
B(s, \sigma) & :=B_{0}+\sum_{i=1}^{\kappa} B_{i} e^{-s \sigma_{i}}, \\
C(s, \nu) & :=C_{0}+\sum_{i=1}^{\kappa} C_{i} e^{-s v_{i}}, \\
\Delta_{G}(s, \tau) & :=s I_{n}-A_{0}-\sum_{i=1}^{\kappa} A_{i} e^{-s \tau_{i}} .
\end{aligned}
$$

Conventionally, $\operatorname{det}\left(\Delta_{G}(s, \tau)\right)$ is called the characteristic function, and $\operatorname{det}\left(\Delta_{G}(s, \tau)\right)=0$ is the characteristic equation, whose zeros are the characteristic roots. Since $\operatorname{det}\left(\Delta_{G}(s, \tau)\right)$ is quasi when $\tau \neq 0$, there are infinitely many characteristic roots. Due to infinite-dimensionality, it is considerably difficult to deal with stability investigation directly in terms of numerically computing the characteristic roots.

We collect properties about the characteristic roots in the following remark, which pave the way for stability analysis and stabilization.

Remark 2. (i) $\operatorname{det}\left(\Delta_{G}(s, \tau)\right)$ is holomorphic; therefore, all characteristic roots are isolated; (ii) there are only finitely many characteristic roots in any vertical strip $\{s \in \mathscr{C}: \alpha<$ $\mathfrak{R}(s)<\beta\}$ of the complex plane, where $\alpha<\beta \in \mathscr{R}$; (iii) for any $\gamma \in \mathscr{R}$ with $|\gamma|<\infty$, only finitely many characteristic roots exist to the right-hand side of the vertical line $\mathfrak{R}(s)=\gamma$, while infinitely many ones are located to the left-hand side of $\mathfrak{R}(s)=\gamma$ [24, p. 9, Corollary 1.9]; (iv) the characteristic roots are continuous with respect to delays and coefficients [24, p. 10, Theorem 1.14]; (v) in any bounded rectangular area $\{s \in \mathscr{C}: \alpha<\Re(s)<\beta, v<\mathfrak{I}(s)<\rho\}=: \mathscr{D}$, where $\alpha<\beta \in \mathscr{R}$ and $v<\rho \in \mathscr{R}$, there are no infinite sequences of characteristic roots that converge to points in $\mathscr{D}$. To see this, we notice that $\operatorname{det}\left(\Delta_{G}(s, \tau)\right)$ is entire over $\mathscr{C} \backslash\{\infty\}$. Hence, if there is an infinite sequence $\left\{s_{i}\right\}_{i=1}^{\infty}$ in which $s_{i} \in \mathscr{D}$ are mutually distinctive, $\operatorname{det}\left(\Delta_{G}\left(s_{i}, \tau\right)\right)=0$ for each $i$, and $\lim _{i \rightarrow \infty} s_{i}=s_{0}$ with $s_{0} \in \mathscr{D}$, then the uniformity theorem tells that $\operatorname{det}\left(\Delta_{G}(s, \tau)\right) \equiv 0$ over $\mathscr{D}$.

Asymptotical and exponential stabilities in (1) are summarized by Definition 3. Proposition 4 gives necessary and 
sufficient conditions for exponential stability, in terms of the characteristic roots and in light of [24].

Definition 3. Let $x(\phi)(\cdot)$ be the null solution of (1) with respect to the initial condition $\phi(t) \in C\left(\left[-\tau_{\max }, 0\right], \mathscr{R}^{n}\right)$, where $\tau_{\max }$ is defined in (2).

(i) The null solution of (1) is asymptotically stable, or simply $\Sigma_{G}$ is asymptotically stable, if, for any $\epsilon>0$, there exist $\delta>0$ and $\phi(t) \in C\left(\left[-\tau_{\max }, 0\right], \mathscr{R}^{n}\right)$ (resp., $\left.\phi(t) \in C^{1}\left(\left[-\tau_{\max }, 0\right], \mathscr{R}^{n}\right)\right)$ with $\|\phi(\cdot)\|_{*}<\delta$ over $t \in\left[-\tau_{\max }, 0\right]$ such that $\|x(\phi)(\cdot)\|_{*}<\epsilon$ as $t \rightarrow \infty$.

(ii) The null solution of (1) is exponentially stable, or simply $\Sigma_{G}$ is exponentially stable, if there exist constants $\alpha>0$ and $\epsilon>0$ such that $\|x(\phi)(\cdot)\|_{*} \leq \alpha e^{-\epsilon t}\|\phi(\cdot)\|_{*}$ over $t \geq 0$ for all $\phi(t) \in C\left(\left[-\tau_{\max }, 0\right], \mathscr{R}^{n}\right)$ (resp., $\left.\phi(t) \in C^{1}\left(\left[-\tau_{\max }, 0\right], \mathscr{R}^{n}\right)\right)$.

Together with Remark 2, asymptotical and exponential stabilities are equivalent whenever LTI time-delayed systems of retarded type are concerned. Hence, only exponential stability conditions are claimed in Proposition 4 explicitly by restating Proposition 1.6 [24].

Proposition 4. The null solution of (1) is exponentially stable if and only if there is $\epsilon>0$ such that $\mathfrak{R}\left(\lambda_{c, i}\right)<-\epsilon$ for each $i$, where $\lambda_{c, i}$ means a root of the characteristic equation $\operatorname{det}\left(\Delta_{G}(s, \tau)\right)=0$.

2.2. Output Feedback Configuration and Return Difference Relationship. By the notation $\Sigma_{C}:\left(\Sigma_{G}, \Sigma_{H}\right)$, we denote an output feedback connection with $\Sigma_{G}$ being the time-delayed system (1) and $\Sigma_{H}$ being an LTI controller, which has the state-space equation

$$
\Sigma_{H}:\left\{\begin{array}{l}
\dot{\zeta}(t)=\Lambda \zeta(t)+\Gamma \mu(t) \\
\eta(t)=\Theta \zeta(t)+\Pi \mu(t),
\end{array}\right.
$$

where $\Lambda \in \mathscr{R}^{p \times p}, \Gamma \in \mathscr{R}^{p \times l}, \Theta \in \mathscr{R}^{m \times p}$, and $\Pi \in \mathscr{R}^{m \times l}$ are constant matrices. Apparently, the transfer function matrix of $\Sigma_{H}$ is

$$
\begin{aligned}
H(s) & =\Theta \Delta_{H}^{-1}(s) \Gamma+\Pi, \\
\Delta_{H}(s) & :=s I_{p}-\Lambda .
\end{aligned}
$$

On the one hand, the open-loop characteristic function is denoted by $P_{o}(s, \tau)$ and satisfies

$$
P_{o}(s, \tau)=\operatorname{det}\left(\Delta_{G}(s, \tau)\right) \operatorname{det}\left(\Delta_{H}(s)\right) .
$$

On the other hand, the state-space equations for the closedloop system $\Sigma_{C}$ are given by

$$
\begin{aligned}
& {\left[\begin{array}{c}
\dot{x}(t) \\
\dot{\zeta}(t)
\end{array}\right]} \\
& \quad=\left[\begin{array}{cc}
A_{0}-B_{0} \Xi \Pi C_{0} & -B_{0} \Xi \Theta \\
\Gamma\left(C_{0}-D \Xi \Pi C_{0}\right) & \Lambda-\Gamma D \Xi \Theta
\end{array}\right]\left[\begin{array}{l}
x(t) \\
\zeta(t)
\end{array}\right]
\end{aligned}
$$

$$
\begin{aligned}
& +\sum_{i}\left[\begin{array}{cr}
A_{i} & 0 \\
0 & 0
\end{array}\right]\left[\begin{array}{r}
x\left(t-\tau_{i}\right) \\
\zeta\left(t-\tau_{i}\right)
\end{array}\right] \\
& +\sum_{i}\left[\begin{array}{rr}
-B_{0} \Xi \Pi C_{i} & 0 \\
\Gamma\left(C_{i}-D \Xi \Pi C_{i}\right) & 0
\end{array}\right]\left[\begin{array}{l}
x\left(t-v_{i}\right) \\
\zeta\left(t-v_{i}\right)
\end{array}\right] \\
& +\sum_{i}\left[\begin{array}{cc}
-B_{i} \Xi \Pi C_{0} & -B_{i} \Xi \Theta \\
0 & 0
\end{array}\right]\left[\begin{array}{l}
x\left(t-\sigma_{i}\right) \\
\zeta\left(t-\sigma_{i}\right)
\end{array}\right] \\
& +\sum_{i} \sum_{k}\left[\begin{array}{cc}
-B_{i} \Xi \Pi C_{k} & 0 \\
0 & 0
\end{array}\right]\left[\begin{array}{l}
x\left(t-\sigma_{i}-v_{k}\right) \\
\zeta\left(t-\sigma_{i}-v_{k}\right)
\end{array}\right] \\
& +\left[\begin{array}{c}
B_{0} \Xi \\
\Gamma D \Xi
\end{array}\right] u(t)+\sum_{i}\left[\begin{array}{c}
B_{i} \Xi \\
0
\end{array}\right] u\left(t-\sigma_{i}\right)
\end{aligned}
$$

$y(t)$

$$
\begin{aligned}
= & {\left[\begin{array}{ll}
C_{0}-D \Xi \Pi C_{0}-D \Xi \Theta
\end{array}\right]\left[\begin{array}{l}
x(t) \\
\zeta(t)
\end{array}\right] } \\
& +\sum_{i}\left[\begin{array}{ll}
C_{i}-D \Xi \Pi C_{i} & 0
\end{array}\right]\left[\begin{array}{l}
x\left(t-v_{i}\right) \\
\zeta\left(t-v_{i}\right)
\end{array}\right]+D \Xi u(t),
\end{aligned}
$$

where $\Xi:=\left(I_{m}+\Pi D\right)^{-1}$. The closed-loop characteristic function $P_{c}(s, \tau, \sigma, \nu)$ is given by

$$
\begin{aligned}
& P_{c}(s, \tau, \sigma, v)=\operatorname{det}\left(\left[\begin{array}{cc}
s I_{n} & 0 \\
0 & s I_{p}
\end{array}\right]\right. \\
& -\left[\begin{array}{cc}
A_{0}-B_{0} \Xi \Pi C_{0} & -B_{0} \Xi \Theta \\
\Gamma\left(C_{0}-D \Xi \Pi C_{0}\right) & \Lambda-\Gamma D \Xi \Theta
\end{array}\right] \\
& -\sum_{i=1}^{\kappa}\left[\begin{array}{cc}
A_{i} & 0 \\
0 & 0
\end{array}\right] e^{-s \tau_{i}}-\sum_{i}\left[\begin{array}{cc}
-B_{0} \Xi \Pi C_{i} & 0 \\
\Gamma\left(C_{i}-D \Xi \Pi C_{i}\right) & 0
\end{array}\right] e^{-s v_{i}} \\
& -\sum_{i=1}^{\kappa}\left[\begin{array}{cc}
-B_{i} \Xi \Pi C_{0} & -B_{i} \Xi \Theta \\
0 & 0
\end{array}\right] e^{-s \sigma_{i}} \\
& \left.-\sum_{i=1}^{\kappa} \sum_{k}\left[\begin{array}{cc}
-B_{i} \Xi \Pi C_{k} & 0 \\
0 & 0
\end{array}\right] e^{-s\left(\sigma_{i}+v_{k}\right)}\right) .
\end{aligned}
$$

By (6) and (8), if $\Sigma_{C}$ is well-posed (i.e., $I_{m}+\Pi D$ is nonsingular), both the open-loop system, denoted by $\Sigma_{O}$, and the closed-loop system $\Sigma_{C}$ are retarded type. Hence, Definition 3 and Proposition 4 apply to $\Sigma_{C}$ as well.

To attack stability in $\Sigma_{C}$, we need to address how to compute $P_{c}(s, \tau, \sigma, v)$. This relates us to the return difference relationship given below. Since the derivation algebras are standard and trivial, we omit the details:

$$
\begin{aligned}
& P_{c}(s, \tau, \sigma, \nu)=\operatorname{det}\left(\Delta_{G}(s, \tau)\right) \\
& \cdot \operatorname{det}\left(\Delta_{H}(s)\right) \frac{\operatorname{det}\left(I_{m}+H(s) G(s, \tau, \sigma, \nu)\right)}{\operatorname{det}\left(I_{m}+\Pi D\right)} .
\end{aligned}
$$


Although the return difference relationship (9) is also in form as what we know in LTI systems, we must be cautious in connecting (9) with the argument principle so as to create stability criteria for the concerned time-delayed systems. Note the following:

(i) Exponentials in delays are involved. Open-loop unstable poles in $\Sigma_{G}$ (i.e., the zeros of $\operatorname{det}\left(\Delta_{G}(s, \tau)\right)=$ $0)$, if any, cannot be calculated simply. $\operatorname{det}\left(\Delta_{G}(s, \tau)\right)=$ 0 has infinitely many zeros; it is almost meaningless to numerically compute unstable poles, if any.

(ii) Since $e^{\alpha s}=1+\alpha s+\alpha^{2} s^{2} / 2 !+\alpha^{3} s^{3} / 3 !+\cdots, \Delta_{G}(s, \tau)$ is a quasi-polynomial; $G(s, \tau, \sigma, \nu)$ is meromorphic in the sense that all its entries are meromorphic. Hence, the argument principle does apply to (9), whenever their right-hand sides vanish nowhere on a contour.

(iii) Reducible factors may exist between $P_{c}(s, \tau, \sigma, \nu)$ and $P_{o}(s, \tau)$; they may stem from the following: (1) input and/or output decoupling zeros in $\Sigma_{G}$ and/or $\Sigma_{H}$, due to uncontrollable and/or unobservable modes [31]; (2) input and/or output decoupling zeros in $\Sigma_{O}$ caused by zero/pole cancellations between $\Sigma_{G}$ and $\Sigma_{H}$, when they are cascaded in $\Sigma_{O}$; and (3) algebraic cancellations, which are poles in $\Sigma_{O}$ that are not affected by feedback or poles that are assigned to locations of other poles of $\Sigma_{O}$ [32]. Removing all reducible factors, if any, not all closed-loop characteristic roots are reflected by (9). Consequently, asymptotical/exponential stability of $\Sigma_{C}$ cannot be examined accordingly. Since $P_{c}(s, \tau, \sigma, \nu)$ and $P_{o}(s, \tau)$ are quasi-polynomials, their coprimeness actually cannot be examined.

\section{Stability Analysis of Time-Delayed Systems}

3.1. Contour, Assumptions, and Problem Formulation. Now we describe a Cauchy contour for utilizing the argument principle of complex analysis in light of $[30,33]$. In Figure 1, the contour is a simply closed curve marked by the dashed line, whose vertical portion is overlapped actually with the imaginary axis. Let us denote by $\operatorname{Int}(\mathcal{N})$ the interior circumscribed by $\mathcal{N}$ to its right. Thus, $\operatorname{Int}(\mathcal{N})$ is an open set and $\operatorname{Int}(\mathscr{N})=\mathscr{C}^{+}$. In the paper, the entry-belonging $s \in \mathcal{N}$ is equivalent to saying $j \omega \in \mathscr{N}$ or the point $j \omega$ is on the imaginary axis; namely, $\omega \in(-\infty, \infty)$. In the cases involving $\epsilon$-exponential stability, we need to use the $\epsilon$-shifted Cauchy contour that is denoted by $\mathcal{N}+\epsilon$ with $\epsilon>0$ being a constant. The exact meaning of the relation $s \in \mathcal{N}+\epsilon$ is $s=j \omega+\epsilon$ with $j \omega \in \mathcal{N}$ or $\omega \in(-\infty, \infty)$.

To deal with the issues explained in Section 2.2 about the return difference equations, we introduce a polynomial $\beta(s)$, namely, the scaling free-term, into (9), and obtain

$$
\begin{aligned}
\frac{P_{c}(s, \tau, \sigma, \nu)}{\beta(s)}= & \frac{\operatorname{det}\left(\Delta_{G}(s, \tau)\right) \operatorname{det}\left(\Delta_{H}(s)\right)}{\beta(s)} \\
& \cdot \frac{\operatorname{det}\left(I_{m}+H(s) G(s, \tau, \sigma, \nu)\right)}{\operatorname{det}\left(I_{m}+\Pi D\right)}
\end{aligned}
$$

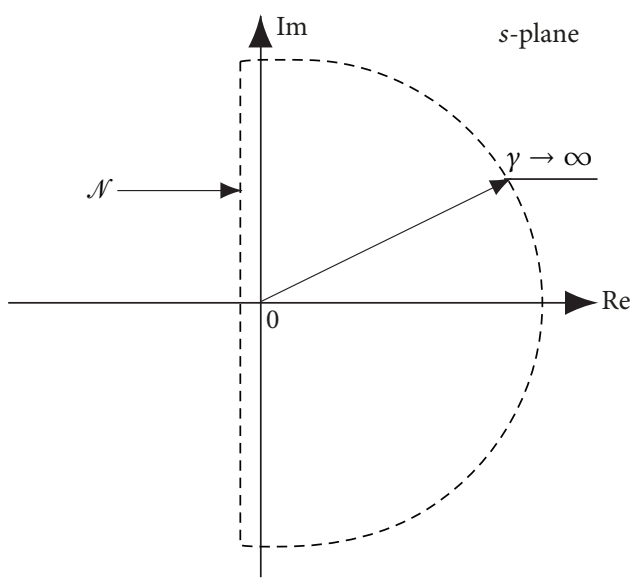

Figure 1: The Cauchy contour $\mathcal{N}$.

which is a generalized return difference relation. By means of $\beta(s), P_{o}(s, \tau)$ and $P_{c}(s, \tau, \sigma, \nu)$ can be handled separately so that characteristic roots distribution of $P_{o}(s, \tau)$ and coprimeness between them are surmounted. About the scaling freeterm, we assume the following.

(A1) $\beta(s)$ is a Hurwitz polynomial with all the roots being far enough from the imaginary axis; thus it has no zeros in $\mathcal{N} \cup \operatorname{Int}(\mathcal{N})$ and $|\beta(0)|$ is as large as desired.

(A2) $\left|\operatorname{det}\left(\Delta_{G}(s, \tau)\right) \operatorname{det}\left(\Delta_{H}(s)\right) / \beta(s)\right|<L$ for some constant $0<L<\infty$ over $s \in \mathcal{N}$.

Obviously, (A1) guarantees that (10) is meromorphic, and $\beta(s)$ vanishes nowhere along $\mathcal{N}$; even if there exists any cancellation between $\beta(s)$ and $P_{c}(s, \tau, \sigma, \nu)$, those cancelled closed-loop characteristic roots must be outside $\mathcal{N} \cup \operatorname{Int}(\mathcal{N})$. By (A1) and (A2), the locus can be plotted within a prespecified bounded area around the origin without prior frequency sweeping. This brings us numerical and graphical convenience. One will understand this by the remarks about Theorem 5.

Our first problem is to establish frequency-domain criteria for testing stability of $\Sigma_{C}$.

\subsection{Complex Scaling Stability Criteria}

Theorem 5. Suppose that the closed-loop time-delayed system $\Sigma_{C}$ is well-posed. Then, $\Sigma_{C}$ is asymptotically stable, if and only if, for some arbitrarily chosen finite-degree polynomial $\beta(s)$ satisfying (A1) and (A2), the generalized locus

$$
\begin{aligned}
\left.f(j \omega, \beta)\right|_{j \omega \in \mathcal{N}} & \frac{\operatorname{det}\left(\Delta_{G}(j \omega, \tau)\right) \operatorname{det}\left(\Delta_{H}(j \omega)\right)}{\beta(j \omega)} \\
& \left.\quad \frac{\operatorname{det}\left(I_{m}+H(j \omega) G(j \omega, \tau, \sigma, \nu)\right)}{\operatorname{det}\left(I_{m}+\Pi D\right)}\right|_{j \omega \in \mathcal{N}}
\end{aligned}
$$

vanishes nowhere, and its number of clockwise encirclements around the origin $(0, j 0)$ is equal to that of counterclockwise ones. The contour $\mathcal{N}$ is defined in Figure 1. 
Proof. As is illustrated in Figure 1, let $j \omega \in \mathcal{N}$ move from a point $j \omega_{0}$ clockwise along $\mathcal{N}$; or we start from $j \omega=j \omega_{0}^{-}$ on $\mathscr{N}$. When we return to $j \omega_{0}$ from the other side along $\mathcal{N}$, we arrive at $j \omega=j \omega_{0}^{+}$. We assert readily by applying the argument principle [30] to (10) that

$$
\begin{aligned}
N & \left(\frac{P_{c}(s, \tau, \sigma, \nu)}{\beta(s)}\right)-P\left(\frac{P_{c}(s, \tau, \sigma, \nu)}{\beta(s)}\right) \\
& =\lim _{j \omega \rightarrow j \omega_{0}^{-} \in \mathcal{N}} L f(j \omega, \beta)-\lim _{j \omega \rightarrow j \omega_{0}^{+} \in \mathcal{N}} L f(j \omega, \beta) \\
& =n_{c e}\left(\left.f(j \omega, \beta)\right|_{j \omega \in \mathcal{N}}\right)-n_{\bar{c} e}\left(\left.f(j \omega, \beta)\right|_{j \omega \in \mathcal{N}}\right),
\end{aligned}
$$

where $N(\cdot)$ and $P(\cdot)$ are the numbers for zeros and poles of $(\cdot)$ in $\operatorname{Int}(\mathscr{N})$ (up to multiplicity), respectively, and $n_{c e}(\cdot)$ and $n_{\bar{c} e}(\cdot)$ are the numbers of clockwise and counterclockwise encirclements of the locus around the origin, respectively. $\angle(\cdot)$ is the complex argument of $(\cdot)$. The last equation of (12) is validated by the fact that the locus $\left.f(j \omega, \beta)\right|_{j \omega \in \mathcal{N}}$ is a closed curve.

It follows by the assumption about $\beta(s)$ that

$$
\begin{aligned}
& N\left(\frac{P_{c}(s, \tau, \sigma, v)}{\beta(s)}\right)=N\left(P_{c}(s, \tau, \sigma, \nu)\right), \\
& P\left(\frac{P_{c}(s, \tau, \sigma, v)}{\beta(s)}\right)=0 .
\end{aligned}
$$

Let $n_{C}$ be the pole number of $\Sigma_{C}$ in $\operatorname{Int}(\mathcal{N})$. It follows that $n_{C}=N\left(P_{c}(s, \tau, \sigma, \nu)\right)$. By Remark 2(iii), $n_{C}$ is a finite integer. Substituting them for (12), we obtain that

$$
\begin{aligned}
n_{C} & =n_{c e}\left(\left.f(j \omega, \beta)\right|_{j \omega \in \mathcal{N}}\right)-n_{\bar{c} e}\left(\left.f(j \omega, \beta)\right|_{j \omega \in \mathcal{N}}\right) \\
& =: n_{n e}\left(\left.f(j \omega, \beta)\right|_{j \omega \in \mathcal{N}}\right)
\end{aligned}
$$

as long as $f(j \omega, \beta)$ vanishes nowhere over $j \omega \in \mathcal{N}$. In (14), $n_{n e}$ means how many net times that the locus $\left.f(j \omega, \beta)\right|_{j \omega \in \mathcal{N}}$ encircles the origin $(0, j 0)$.

Equipped with (14), we are ready to show the main assertion.

To see sufficiency, we see that, under the assumptions on $\beta(s)$ and the locus $\left.f(j \omega, \beta)\right|_{j \omega \in \mathscr{N}}$, (14) can be equivalently interpreted as

$$
\begin{aligned}
n_{C} & =n_{c e}\left(\left.f(j \omega, \beta)\right|_{j \omega \in \mathcal{N}}\right)-n_{\bar{c} e}\left(\left.f(j \omega, \beta)\right|_{j \omega \in \mathcal{N}}\right) \\
& =n_{n e}\left(\left.f(j \omega, \beta)\right|_{j \omega \in \mathcal{N}}\right)=0
\end{aligned}
$$

which says by Proposition 4 that $\Sigma_{C}$ is asymptotically stable.

To see necessity, assume that $\Sigma_{C}$ is asymptotically stable. Hence, no closed-loop characteristic root belongs to $\mathcal{N} \cup$ $\operatorname{Int}(\mathcal{N})$. To complete the proof, it remains to show that some Hurwitz polynomial $\beta(s)$ can be constructed such that (A1) and (A2) are satisfied, and the corresponding locus vanishes nowhere over $j \omega \in \mathcal{N}$, and $n_{c e}(\cdot)=n_{\overline{c e}}(\cdot)$.

Firstly, by the stability assumption about $\Sigma_{C}$, if $s_{0}$ is a zero of $P_{c}(s, \tau, \sigma, v)$, namely, $P_{c}\left(s_{0}, \tau, \sigma, v\right)=0$, then it the fact that $\mathfrak{R}\left(s_{0}\right)<0$ holds. Thus, $P_{c}(j \omega, \tau, \sigma, \nu) \neq 0$ over $j \omega \in \mathcal{N}$.
Secondly, recalling the definition for determinants, we can write that

$$
\begin{aligned}
P_{c}(s, \tau, \sigma, \nu)= & s^{n+p}+a_{n+p-1}(s, \tau, \sigma, \nu) s^{n+p-1}+\cdots \\
& +a_{0}(s, \tau, \sigma, \nu)
\end{aligned}
$$

where $\left\{a_{i}(s, \tau, \sigma, \nu)\right\}$ consists of functions in terms of $e^{-s \tau}, e^{-s \sigma}$, $e^{-s(\tau+\sigma, \nu)}$, and their products as appropriately. Bearing this in mind, it is not hard to show that

$$
\left|a_{i}(j \omega, \tau, \sigma, v)\right|<L^{\prime}, \quad \forall j \omega \in \mathcal{N}
$$

for some $0<L^{\prime}<\infty$ and uniformly for each $i$.

Thirdly, we construct a scaling in form of $\beta(s)=\prod_{i=1}^{n+p}(s+$ $\left.\lambda_{r, i}\right)$, where $\mathfrak{R}\left(\lambda_{r, i}\right)<-\epsilon$; or $\beta(s)$ has no zeros in $\mathcal{N} \cup \operatorname{Int}(\mathcal{N})$; thus, (A1) is satisfied. It is straightforward to see that

$$
\begin{gathered}
\frac{P_{c}(j \omega, \tau, \sigma, \nu)}{\beta(j \omega)} \neq 0, \quad \forall j \omega \in \mathcal{N}, \\
\left|\frac{P_{c}(j \omega, \tau, \sigma, \nu)}{\beta(j \omega)}\right|<L, \quad \forall j \omega \in \mathcal{N}
\end{gathered}
$$

for some $0<L<\infty$, say $L=\sup _{j \omega \in \mathcal{N}}\left|P_{c}(j \omega, \tau, \sigma, \nu) / \beta(s)\right|+\mu$ with $\mu>0$ being arbitrarily given. Clearly, $\sup _{j \omega \in \mathscr{N}} \mid P_{c}(j \omega$, $\tau, \sigma, \nu) / \beta(j \omega) \mid$ makes sense by $(17), \operatorname{deg}\left(P_{c}(s, \tau, \sigma, \nu)\right)=n+p$, and $\operatorname{deg}(\beta(s))=n+p$. Thus, (A2) is satisfied with $\beta(s)$.

Finally, based on $\beta(s)$ so constructed, write $P_{c}(s, \tau, \sigma, \nu) /$ $\beta(s)=f(s, \beta)$, which satisfies (10), and consequently the argument principle relationship (14) is true. This, after repeating the sufficiency arguments but in the reverse direction, implies that the locus $\left.f(j \omega, \beta)\right|_{j \omega \in \mathcal{N}}$ vanishes nowhere with respect to $j \omega \in \mathcal{N}$, and $0=n_{C}=n_{n e}$. Accordingly, $n_{c e}=$ $n_{\bar{c} e}$.

Now we collect remarks about Theorem 5.

(i) The conditions are necessary and sufficient, dependent on time delays, independent of any openloop structural features, and stated via generalized frequency-domain features. In addition, the locus encirclement and orientation can be self-defined. The results apply to open-loop systems by plotting $\Delta_{G}(s, \tau) /\left.\beta(s)\right|_{s \in \mathcal{N}}$. This reduces to the idea of [29].

(ii) Note that the scaling $\beta(s)$ is not unique. Actually, $\beta(s)$ can be arbitrary as long as it is a Hurwitz polynomial. However, as graphical implementation is concerned, it is prudent to have $\operatorname{deg}(\beta(s))=n+p$. If $\operatorname{deg}(\beta(s))>$ $n+p$, the locus may approach the origin numerically as $\omega \rightarrow \infty$, and this makes it difficult to count encirclements, whereas if $\operatorname{deg}(\beta(s))<n+p$, the corresponding locus may contain portions at infinity as $\omega \rightarrow \infty$.

Together with (A1), $\operatorname{deg}(\beta(s))=n+p$ guarantees that the whole locus can be plotted within a bounded region around the origin as compactly as possible by choosing the roots of $\beta(s)$ to be located far away sufficiently to the left of the imaginary axis. In view of this, no frequency sweeping is prerequisite for correctly testing the conditions. 
(iii) When using the $\epsilon$-shifted Cauchy contour $\mathcal{N}+$ $\epsilon$, possible $\epsilon$-exponential stability of the concerned time-delayed system can be reflected, that is, if all the closed-loop poles are at the left-hand side of the vertical line $\operatorname{Re}(s)=\epsilon$. Also, if $\Sigma_{H}$ and/or $\Sigma_{G}$ have any poles on the imaginary axis, we need the contour $\mathcal{N}+\epsilon$ to detour such poles and guarantee numerical welldefinedness of the right-hand side of (10). It should be stressed that analytically there is no need to use the shifted contour even if there are imaginary poles.

(iv) Though the conditions are stated in a graphical manner for stability testing and robustness evaluation as known in LTI continuous-time systems, they can be interpreted and implemented purely in a numerical way. Indeed, the criterion conditions can be examined via computing the argument incremental, namely, $\left.\nabla \angle f(j \omega, \beta)\right|_{\omega \in(-\infty, \infty)}$, of $f(j \omega, \beta)$ along the imaginary axis, and checking if $|\nabla \angle f(j \omega, \beta)|_{\omega \in(-\infty, \infty)} \mid \leq \eta$ (where $\eta>0$ is a small number as tolerance error), while neither loci plotting nor assumptions checking is involved.

A self-evident corollary of Theorem 5 is as follows.

Corollary 6. Suppose that the closed-loop time-delayed system $\Sigma_{C}$ is well-posed. Also assume that the open-loop system is asymptotically stable. Then, $\Sigma_{C}$ is asymptotically stable, if and only if the locus $\left.\operatorname{det}\left(I_{m}+H(j \omega) G(j \omega, \tau, \sigma, v)\right)\right|_{j \omega \in \mathcal{N}}$ vanishes nowhere, and its number of clockwise encirclements around the origin $(0, j 0)$ is equal to that of counterclockwise ones.

Proof. Since the open-loop system is asymptotically stable, it follows that the open-loop characteristic polynomial $\operatorname{det}\left(\Delta_{G}(s, \tau)\right) \operatorname{det}\left(\Delta_{H}(s)\right)=P_{o}(s, \tau)$ is a Hurwitz polynomial. Let $\beta(s)=P_{o}(s, \tau)$ and note that $\operatorname{det}\left(I_{m}+\Pi D\right) \neq 0$ is a constant. The desired assertion follows from Theorem 5 .

Corollary 6 implies that the conventional Nyquist loci are merely special cases of the generalized ones. The former generally reveal partially the frequency-domain features of a concern system, which may or may not be adequate or complete in reflecting stability and robustness.

\section{Stabilization of Time-Delayed Systems}

4.1. Problem Formulation with Time-Delayed State Feedback. Let us consider the time-delayed system

$$
\dot{x}(t)=A_{0} x(t)+\sum_{i=1}^{\kappa} A_{i} x\left(t-\tau_{i}\right)+B_{0} u(t),
$$

where the matrices and vectors are the same as those in (1). To differential-difference state-space equation (19), we introduce the state feedback

$$
u(t)=-\left[K_{0} x(t)+\sum_{i=1}^{\kappa} K_{i} x\left(t-\tau_{i}\right)\right]+v(t)
$$

where $v(t) \in \mathscr{R}^{m}$ is a new reference input and $K_{i} \in \mathscr{R}^{m \times n}, i=$ $0,1, \ldots, \kappa$, are static feedback gains. Then, the closed-loop system can be described by

$$
\begin{aligned}
\dot{x}(t)= & {\left[A_{0}-B_{0} K_{0}\right] x(t)+\sum_{i=1}^{\kappa}\left[A_{i}-B_{0} K_{i}\right] x\left(t-\tau_{i}\right) } \\
& +B_{0} v(t)
\end{aligned}
$$

and the associated closed-loop characteristic polynomial is

$$
\begin{aligned}
& P_{c}(s, \tau) \\
& \quad=\operatorname{det}\left(s I_{n}-\left[A_{0}-B_{0} K_{0}\right]-\sum_{i=1}^{\kappa}\left[A_{i}-B_{0} K_{i}\right] e^{-s \tau_{i}}\right) .
\end{aligned}
$$

The characteristic polynomial $P_{c}(s, \tau)$ for $(21)$ can be rewritten as

$$
\begin{aligned}
& P_{c}(s, \tau)=\operatorname{det}\left(s I_{n}-A_{0}+B_{0} K_{0}\right) \cdot \operatorname{det}\left(I_{n}\right. \\
& \left.-\left(s I_{n}-A_{0}+B_{0} K_{0}\right)^{-1} \sum_{i=1}^{\kappa}\left[A_{i}-B_{0} K_{i}\right] e^{-s \tau_{i}}\right) \\
& \quad=P_{0}(s) \operatorname{det}\left(I_{n}\right. \\
& \left.\quad-\Delta^{-1}\left(s, K_{0}\right) \sum_{i=1}^{\kappa}\left[A_{i}-B_{0} K_{i}\right] e^{-s \tau_{i}}\right),
\end{aligned}
$$

where $P_{0}(s)=\operatorname{det}\left(\Delta\left(s, K_{0}\right)\right)$ and $\Delta\left(s, K_{0}\right)=s I_{n}-A_{0}+B_{0} K_{0}$. Clearly, $P_{0}(s)$ is the closed-loop characteristic polynomial of the LTI system $\dot{x}=A_{0} x+B_{0} u$ subject to the state feedback $u=K_{0} x$.

Hence, the stabilization problem is formulated as follows: design the static feedback gains $K_{i} \in \mathscr{R}^{m \times n}, i=0,1, \ldots, \kappa$ such that all the roots of $P_{c}(s, \tau)=0$ possess negative real parts.

4.2. Stabilization via Time-Delayed Dynamics Cancellation. In the control theory, stabilization in LTI systems by static state feedback has been well addressed. In view of the specific expression of the closed-loop time-delayed system (21), we see that if the time-delayed states can be cancelled by the state feedback, then the closed-loop system reduces to an LTI one, which can be stabilized by means of some pole assignment as usual.

Proposition 7. Consider time-delayed system (19), to which the static state feedback (20) is introduced. Also, suppose that the pair $\left(A_{0}, B_{0}\right)$ is stabilizable and each algebraic equation $A_{i}=B_{0} K_{i}$ is solvable in terms of $K_{i}$ for each $i=1, \ldots, \kappa$. Then, there exists a static feedback gain sequence $\left\{K_{i}\right\}_{i=0,1, \ldots, \kappa}$ such that closed-loop system (21) is asymptotically stable.

Proof. Under the solvability assumption about $A_{i}=B_{0} K_{i}$, there are $K_{i}$ with $i=1, \ldots, \kappa$ such that $A_{i}-B_{0} K_{i}=0$ and 
thus the closed-loop characteristic polynomial $P_{c}(s, \tau)$ turns out to be $P_{c}(s, \tau)=\operatorname{det}\left(s I_{n}-A_{0}+B_{0} K_{0}\right)$. Note that $\left(A_{0}, B_{0}\right)$ is stabilizable. It follows that all the roots of $P_{c}(s, \tau)$ can be assigned to left half-plane by choosing $K_{0}$ as desired.

Proposition 7 is significant merely in the theoretical sense and applies to extremely special time-delayed systems. However, the time-delayed states cancellation idea is inspiring; that is, if the impact of the time-delayed states can be reduced in some sense, then stabilization of the original time-delayed system is achievable by working with a corresponding LTI system in the sense of perturbation analysis. This is what we do in the subsequent subsections.

4.3. Complex Scaling Stabilization via Pole Assignment. To facilitate our following statements, let us define

$$
\begin{aligned}
& g(\beta, s)=\beta^{-1}(s) P_{0}(s) \\
& \cdot \operatorname{det}\left(I_{n}-\Delta^{-1}\left(s, K_{0}\right) \sum_{i=1}^{\kappa}\left[A_{i}-B_{0} K_{i}\right] e^{-s \tau_{i}}\right),
\end{aligned}
$$

where $\beta(s)$ is a prescribed Hurwitz polynomial. Clearly, $g(\beta, s)=\beta^{-1}(s) P_{c}(s, \tau)$.

Now let us examine what happens to $g(\beta, s)$ as the variable $s$ travels along the Cauchy contour $\mathcal{N}$. We observe the following facts:

(i) If the pair $\left(A_{0}, B_{0}\right)$ is completely controllable, the eigenvalues of $A_{0}-B_{0} K_{0}$ can always be assigned arbitrarily on the left half-plane such that $P_{0}(s)$ is a Hurwitz polynomial.

(ii) With respect to some given feedback gains $\left\{K_{i}\right\}_{i=1, \ldots, \kappa}$ and eigenvalues $\left\{\lambda_{k}\right\}_{k=1, \ldots, n}$ that are sufficiently far left to the imaginary axis, it is always possible to determine $K_{0}$ by some poles assignment algorithm such that $P_{0}(s)$ is a Hurwitz polynomial and

$$
\left\|\Delta^{-1}\left(K_{0}, j \omega\right) \sum_{i=1}^{\kappa}\left[A_{i}-B_{0} K_{i}\right] e^{-j \omega \tau_{i}}\right\| \longrightarrow 0,
$$

$\forall j \omega \in \mathcal{N}$.

Based on the above observations, we claim the following results for stabilization.

Theorem 8. Consider time-delayed system (19), to which the static state feedback (20) is introduced. Let the pair $\left(A_{0}, B_{0}\right)$ be controllable. If there exists a feedback gain sequence $\left\{K_{i}\right\}_{i=0,1, \ldots, \kappa}$ satisfying (25) and $A_{0}-B_{0} K_{0}$ is a Hurwitz matrix (all its eigenvalues have negative real parts), then closed-loop system (21) is asymptotically stabilized.

Proof. Under the given assumptions, there exists a feedback gain sequence $\left\{K_{i}\right\}_{i=0,1, \ldots, \kappa}$ such that both of the above two observations are satisfied. Bearing this in mind, let $\beta(s)=$ $P_{0}(s)$, which is a Hurwitz polynomial according to our poles assignment with $K_{0}$. Then, we can use the following generalized locus with respect to the contour $\mathcal{N}$ for stability analysis

$$
\begin{aligned}
& \left.g(\beta, j \omega)\right|_{j \omega \in \mathcal{N}}=\operatorname{det}\left(I_{n}\right. \\
& \left.\quad-\Delta^{-1}\left(K_{0}, j \omega\right) \sum_{i=1}^{\kappa}\left[A_{i}-B_{0} K_{i}\right] e^{-j \omega \tau_{i}}\right)\left.\right|_{j \omega \in \mathcal{N}} .
\end{aligned}
$$

Then, the stabilization is attainable if we can assert that the corresponding locus does not encircle the origin under the given $\left\{K_{i}\right\}_{i=0,1, \ldots, \kappa}$. Or equivalently, a static state feedback (20) with $\left\{K_{i}\right\}_{i=0,1, \ldots, \kappa}$ exists such that all the roots of $P_{c}(s, \tau)=0$ are assigned to the left half-plane.

To this end, let us simply denote the $i$ th eigenvalue by

$$
\lambda_{i}(j \omega)=: \lambda_{i}\left(\Delta^{-1}\left(K_{0}, j \omega\right) \sum_{i=1}^{\kappa}\left[A_{i}-B_{0} K_{i}\right] e^{-j \omega \tau_{i}}\right)
$$

with $i=1, \ldots, n$. Next, it is also clear that, for any specific $j \omega \in \mathcal{N}$,

$$
\lim _{\left\|K_{0}\right\| \rightarrow \infty} \Delta^{-1}\left(K_{0}, j \omega\right) \sum_{i=1}^{\kappa}\left[A_{i}-B_{0} K_{i}\right] e^{-j \omega \tau_{i}}=0 .
$$
that

Using $\left\{\lambda_{i}(j \omega)\right\}_{i=1, \ldots, n}$, we notice by the determinant theory

$$
g(\beta, j \omega)=\prod_{i=1}^{n}\left(1-\lambda_{i}(j \omega)\right)
$$

from which the following points follow immediately.

Firstly, together with the fact that $\Delta^{-1}\left(s, K_{0}\right) \sum_{i=1}^{\kappa}\left[A_{i}-\right.$ $\left.B_{0} K_{i}\right] e^{-s \tau_{i}}$ is continuous in $K_{0}$ and $s$, we can conclude from (25) that $\left|\lambda_{i}(j \omega)\right|<\kappa$ uniformly over $j \omega \in \mathcal{N}$ for some $0<$ $\kappa<1$ sufficiently small. Then, $g(\beta, j \omega) \neq 0$ for all $j \omega \in \mathcal{N}$, which means that the locus $\left.g(\beta, j \omega)\right|_{j \omega \in \mathcal{N}}$ vanishes nowhere as $j \omega$ runs over the contour $\mathcal{N}$; or, equivalently, $g(\beta, j \omega) \neq 0$ over $j \omega \in \mathcal{N}$.

Secondly, $\lim _{\omega \rightarrow \pm \infty} g(\beta, j \omega)=1$ and $\lim _{\left\|K_{0}\right\| \rightarrow \infty} g(\beta, j \omega)$ $=1$ for each fixed $j \omega \in \mathcal{N}$ hold. This implies that, for any $j \omega \in$ $\mathcal{N}$, each complex phaser $\left(1-\lambda_{i}(j \omega)\right)$ must be a complex vector from the origin $(0, j 0)$ to a point in some closed $\kappa$-related neighborhood around $(1, j 0)$, whose complex argument $\angle(1-$ $\left.\lambda_{i}(j \omega)\right)$, which is the angle subtended by the phaser itself and the positive real axis, satisfies $\left|\angle\left(1-\lambda_{i}(j \omega)\right)\right|<2 \pi / n$ uniformly over $j \omega \in \mathcal{N}$ if $\left\|\Delta^{-1}\left(K_{0}, j \omega\right) \sum_{i=1}^{\mathcal{K}}\left[A_{i}-B_{0} K_{i}\right] e^{-j \omega \tau_{i}}\right\| \rightarrow$ 0 for all $j \omega \in \mathcal{N}$. Then, we conclude that

$$
|\angle(g(\beta, j \omega))|=\left|\sum_{i=1}^{n} \angle\left(1-\lambda_{i}(j \omega)\right)\right|<2 \pi,
$$

$\forall j \omega \in \mathcal{N}$.

This says exactly nothing but that the entire locus $g(\beta$, $j \omega)\left.\right|_{j \omega \in \mathcal{N}}$ must be within a bounded range around the point $(1, j 0)$ that excludes the origin $(0, j 0)$, when $\left\|K_{0}\right\|$ is sufficiently large and all eigenvalues of $A_{0}-B_{0} K_{0}$ have negative 
real parts. It follows that the entire locus $\left.g(\beta, j \omega)\right|_{j \omega \in \mathcal{N}}$ has no encirclements around the origin.

Based on the above observations and the argument principle, it follows that the closed-loop characteristic polynomial $P_{c}(s, \tau)$ cannot have zeros in the closed right half-plane. This completes the proof arguments eventually.

Some remarks about Theorem 8 are as follows:

(i) Stabilization in Theorem 5 is meant in asymptotical stability. If the state decreasing ratio is expected, the stabilization design should be constructed in exponential stability. It is simple to see that such stabilization design can be achieved by replacing the contour $\mathcal{N}$ with some $\epsilon$-shifted one, that is, $\mathcal{N}+\epsilon$, with $\epsilon>0$ being an expected decreasing ratio.

(ii) Compared to Proposition 7, the controllability assumption about the pair $\left(A_{0}, B_{0}\right)$ cannot be relaxed to a stabilizability one in general. Otherwise, some eigenvalues of $A_{0}-B_{0} K_{0}$ may not be able to be positioned as appropriately to satisfy the robustness inequality (25).

(iii) In principle, the time-delay-related static feedback gains $\left\{K_{i}\right\}_{i=1, \ldots, \kappa}$ can be taken arbitrarily. However, as far as practical controller implementation is concerned, $\left\|K_{i}\right\|, i=0,1, \ldots, \kappa$, should be reasonable in magnitude, that is, neither too big nor too small. To reflect this concern, it is plausible to take $\left\{K_{i}\right\}_{i=1, \ldots, \kappa}$ such that $\left\|A_{i}-B_{0} K_{i}\right\| \rightarrow \min [34]$ and thus $\left\|K_{0}\right\|$ can be more reasonable in magnitude to satisfy robustness inequality (25).

(iv) A stabilization design procedure is suggested as follows. Step 1. Prescribe $\left\{K_{i}\right\}_{i=1, \ldots, \kappa}$ and compute $\sum_{i=1, \ldots, \kappa}\left\|A_{i}-B_{0} K_{i}\right\|$. Step 2. Prescribe expected eigenvalue candidates $\left\{\lambda_{i}\right\}_{i=1, \ldots, \kappa}$ with $\operatorname{Re}\left(\lambda_{i}\right)<0$. Step 3 . Determine $K_{0}$ via some pole assignment algorithm such that $A_{0}-B_{0} K_{0}$ possesses the expected eigenvalues and satisfies

$\sup _{j \omega \in \mathcal{N}}\left\{\left\|\Delta^{-1}\left(K_{0}, j \omega\right)\right\|\right\} \ll\left\{\sum_{i=1}^{\kappa}\left\|A_{i}-B_{0} K_{i}\right\|\right\}^{-1}$.

It is simple to see that inequality (25) is satisfied as long as (31) is true. As a matter of fact, the following holds for each $\omega \in(-\infty, \infty)$ :

$$
\begin{aligned}
& \left\|\Delta^{-1}\left(K_{0}, j \omega\right) \sum_{i=1}^{\kappa}\left[A_{i}-B_{0} K_{i}\right] e^{-j \omega \tau_{i}}\right\| \\
& \leq\left\|\Delta^{-1}\left(K_{0}, j \omega\right)\right\| \cdot\left\|\sum_{i=1}^{\kappa}\left[A_{i}-B_{0} K_{i}\right] e^{-j \omega \tau_{i}}\right\| \\
& \left\|\sum_{i=1}^{\kappa}\left[A_{i}-B_{0} K_{i}\right] e^{-j \omega \tau_{i}}\right\| \leq \sum_{i=1}^{\kappa}\left\|A_{i}-B_{0} K_{i}\right\| \\
& \sup _{j \omega \in \mathscr{N}}\left\{\left\|\Delta^{-1}\left(K_{0}, j \omega\right)\right\|\right\} \geq\left\|\Delta^{-1}\left(K_{0}, j \omega\right)\right\| .
\end{aligned}
$$

In addition, it is well known that $\sup _{j \omega \in \mathscr{N}}\left\{\| \Delta^{-1}\left(K_{0}\right.\right.$, $j \omega) \|\}$ can be manipulated arbitrarily by using the $H_{\infty}$ norm performance optimization technique [35].

The so-designed static feedback gains sequence $\left\{K_{i}\right\}_{i=0,1, \ldots, \kappa}$ has nothing to do with the time delays in the state and control input. In view of this, we say that the suggested stabilization is robust with respect to the time delays in the state and control input.

(v) With some rough words and abuse of terminologies, the stabilization design consists of two design procedures: firstly, the embedding LTI differential equation $\dot{x}=A_{0} x+B_{0} u$ is stabilized as a continuoustime subsystem; secondly, the embedding difference relations in terms of the time-delayed states are stabilized as a discrete-time subsystem. In this sense, the suggested stabilization technique is hybrid and quite intuitive.

4.4. Stabilization Interpreted with Small-Gain Theorem. In this subsection, we show that some stabilization results similar to those of Theorem 8 follow readily by the small-gain theorem $[36,37]$ under the inequality condition

$$
\max _{\omega \in(-\infty, \infty)}\left\|\Delta^{-1}\left(K_{0}, j \omega\right)\right\|\left(\sum_{i=1}^{\kappa}\left\|A_{i}-B_{0} K_{i}\right\|\right)<1 .
$$

To this end, let us note that closed-loop time-delayed system (21) can be equivalently interpreted as a feedback configuration in form of

$$
\begin{aligned}
& \dot{x}(t)=\left[A_{0}-B_{0} K_{0}\right] x(t)+z(t)+B_{0} v(t), \\
& y(t)=x(t), \\
& z(t)=\sum_{i=1}^{\kappa}\left[A_{i}-B_{0} K_{i}\right] y\left(t-\tau_{i}\right),
\end{aligned}
$$

where $z(t)=\sum_{i=1}^{\kappa}\left[A_{i}-B_{0} K_{i}\right] x\left(t-\tau_{i}\right)$ is viewed as a static state feedback. Similar feedback connection remodeling in time-delayed systems can be found in [10], for dealing with absolute stability in some Lur'e problem settings.

We observe by the Parseval theorem that

$$
\begin{aligned}
& \|z(\cdot)\|_{L_{2}[0, \infty)}=\left\|\sum_{i=1}^{\kappa}\left[A_{i}-B_{0} K_{i}\right] y\left(\cdot-\tau_{i}\right)\right\|_{L_{2}[0, \infty)} \\
& \leq \sum_{i=1}^{\kappa}\left\|\left[A_{i}-B_{0} K_{i}\right] y\left(\cdot-\tau_{i}\right)\right\|_{L_{2}[0, \infty)} \\
& =\sum_{i=1}^{\kappa}\left(\int_{0}^{\infty}\left\|\left[A_{i}-B_{0} K_{i}\right] y\left(\sigma-\tau_{i}\right)\right\|^{2} d \sigma\right)^{\frac{1}{2}} \\
& =\sum_{i=1}^{\kappa}\left(\frac{1}{2 \pi} \int_{-\infty}^{\infty}\left(\left[A_{i}-B_{0} K_{i}\right] e^{-j \omega \tau_{i}} Y(j \omega)\right)^{*}\right. \\
& \left..\left(\left[A_{i}-B_{0} K_{i}\right] e^{-j \omega \tau_{i}} Y(j \omega)\right) d \omega\right)^{\frac{1}{2}}=\sum_{i=1}^{\kappa}\left(\frac{1}{2 \pi}\right. \\
& \left.. \int_{-\infty}^{\infty}\left\|\left[A_{i}-B_{0} K_{i}\right] e^{-j \omega \tau_{i}} Y(j \omega)\right\|^{2} d \omega\right)^{\frac{1}{2}}
\end{aligned}
$$




$$
\begin{aligned}
& \leq \sum_{i=1}^{\kappa}\left(\frac{1}{2 \pi} \int_{-\infty}^{\infty}\left\|\left[A_{i}-B_{0} K_{i}\right]\right\|^{2}\|Y(j \omega)\|^{2} d \omega\right)^{\frac{1}{2}} \\
& =\sum_{i=1}^{\kappa}\left(\left\|\left[A_{i}-B_{0} K_{i}\right]\right\|^{2} \cdot \frac{1}{2 \pi} \int_{-\infty}^{\infty}\|Y(j \omega)\|^{2} d \omega\right)^{\frac{1}{2}} \\
& =\sum_{i=1}^{\kappa}\left\|\left[A_{i}-B_{0} K_{i}\right]\right\|\left(\int_{0}^{\infty}\|y(\sigma)\|^{2} d \sigma\right)^{\frac{1}{2}} \\
& =\left(\sum_{i=1}^{\kappa}\left\|\left[A_{i}-B_{0} K_{i}\right]\right\|\right)\|y(\cdot)\|_{L_{2}[0, \infty)} .
\end{aligned}
$$

In the above, $\|x(\cdot)\|_{L_{2}[0, \infty)}$ denotes the $L_{2}$-function norm of $x(t)$.

Now let us assume that $v(t) \equiv 0$ for all $t$. It follows that

$$
\sup _{\|y(\cdot)\|_{L_{2}[0, \infty)} \neq 0}\left\{\frac{\|z(\cdot)\|_{L_{2}[0, \infty)}}{\|y(\cdot)\|_{L_{2}[0, \infty)}}\right\} \leq \sum_{i=1}^{\kappa}\left\|\left[A_{i}-B_{0} K_{i}\right]\right\| .
$$

Furthermore, note that LTI system (34) with $v(t) \equiv 0$ satisfies

$$
\begin{aligned}
& \sup _{\|z(\cdot)\|_{L_{2}[0, \infty)} \neq 0}\left\{\frac{\|y(\cdot)\|_{L_{2}[0, \infty)}}{\|z(\cdot)\|_{L_{2}[0, \infty)}}\right\} \\
& =\max _{\omega \in(-\infty, \infty)}\left\|\Delta^{-1}\left(K_{0}, j \omega\right)\right\|
\end{aligned}
$$

which is exactly the $H_{\infty}$ norm defined between $z(\cdot)$ and $y(t)$ of (34); or the $L_{2}$-gain from $z(\cdot)$ to $y(\cdot)$ is equivalent to its operator norm induced by the $L_{2}$-function norm. This is a well-known result about LTI systems in robust performance [35].

Based on (33), (36), and (37), the small-gain theorem $[36,37]$ introduces to us the following stabilizability result immediately but in the $L_{2}$-stability sense.

Theorem 9. Consider time-delayed system (19), to which the static state feedback (20) is introduced. Let the pair $\left(A_{0}, B_{0}\right)$ be controllable. If there exists a feedback gain sequence $\left\{K_{i}\right\}_{i=0,1, \ldots, k}$ satisfying (33) and $A_{0}-B_{0} K_{0}$ is a Hurwitz matrix, then the closed-loop system (21) is $L_{2}$-stabilized.

Some remarks about Theorem 9 are as follows:

(i) At first glance, the same assumptions are seemingly supposed for guaranteeing stabilization. In Theorem 8, stability of $A_{0}-B_{0} K_{0}$ is for the closed-loop characteristic polynomial $P_{c}(s, \tau)$ to be reexpressed such that generalized locus formula (26) is welldefined for applying the argument principle, while in Theorem 9 stability of $A_{0}-B_{0} K_{0}$ is for welldefinedness of the $H_{\infty}$-norm of $\Delta^{-1}\left(s, K_{0}\right)$.

(ii) Note that (31) yields (33) to us. Interpreting this in light of Theorem 9, one can easily see that the stabilization procedure suggested in the remarks for Theorem 8 can also be adopted for stabilization design in the $L_{2}$-stability sense.

(iii) Inequality (31) implies (33) readily; however, the reverse may not be true. This tells us that the smallgain condition may be less conservative than that of (31). It is stressed that the relationships between (25) and (33) are not definite.

(iv) Since the measured output of (34) is exactly the state vector $x(t)$ of closed-loop time-delayed system (21), therefore, $L_{2}$-stability of the feedback connection formed by (34) and $z(t)=\sum_{i=1}^{\kappa}\left[A_{i}-B_{0} K_{i}\right] x\left(t-\tau_{i}\right)$ implies that $\|x(t)\| \rightarrow 0$ as $t \rightarrow \infty$. That is, asymptotical stability of closed-loop time-delayed system (21) can be asserted if $L_{2}$-stability of the equivalent feedback connection is confirmed.

\section{Numerical Illustrations}

5.1. Stability Analysis Illustrations. Stability of the following scalar linear time-delayed system of retarded type is considered:

$$
\Sigma_{G}:\left\{\begin{array}{l}
\dot{x}(t)=\left[\begin{array}{cc}
-0.5 & 0 \\
1 & -4
\end{array}\right] x(t)+\left[\begin{array}{cc}
0.25 & 0 \\
1.5 & -2.4
\end{array}\right] x(t-\tau)+\left[\begin{array}{l}
1 \\
0
\end{array}\right] u(t)+\left[\begin{array}{c}
0 \\
0.2
\end{array}\right] u(t-\sigma), \\
y(t)=\left[\begin{array}{ll}
-0.5 & 1
\end{array}\right] x(t)+\left[\begin{array}{ll}
1 & 0
\end{array}\right] x(t-\tau),
\end{array}\right.
$$

where $\tau>0$ is the time delay in the states, and $\sigma>0$ is that in the control input.

To $\Sigma_{G}$, implement a static output feedback $u(t)=-K y(t)$ with $K$ being constant; namely, $\Sigma_{H}: H(s)=K$ in the feedback connection of $\Sigma_{C}$. In numeric computation, the contour $\mathcal{N}$ is used, while the Hurwitz polynomial $\beta(s)=$ $(s+30)^{2}$ is chosen.
5.1.1. Stability Analysis When $K$ Varies. Now we investigate stability with $K \in[-4,4]$, while $\tau=0.2$ and $\sigma=0.3$ are fixed. The generalized loci are plotted in Figure 2 with $K \in\{-4,-2,0,2,4\}$, respectively. The $N$ numbers in the legend box are the net encirclements around the origin of the loci, which are obtained by complex argument incremental computation as explained in the remarks about Theorem 5 . 


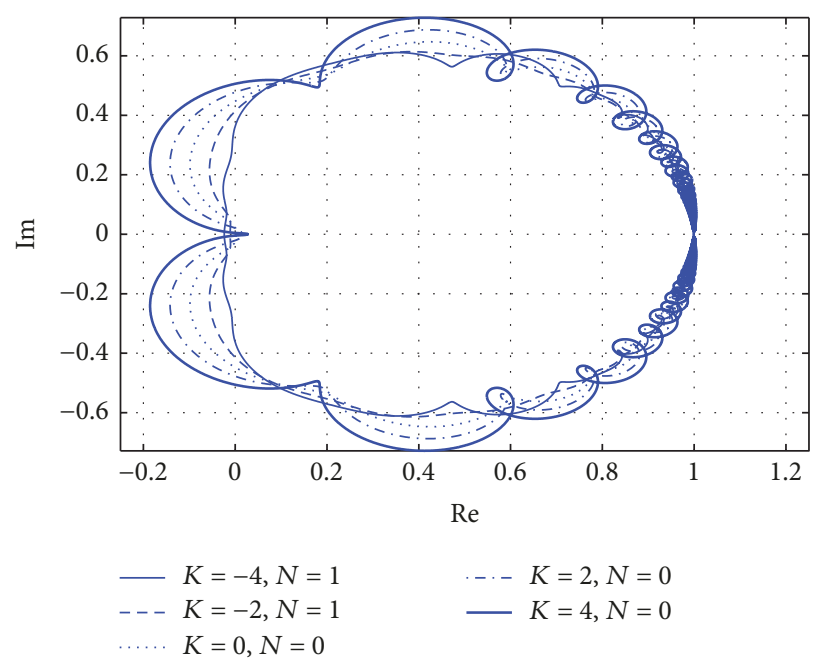

Figure 2: Generalized loci under the feedback gain $K \in[-4,4]$.

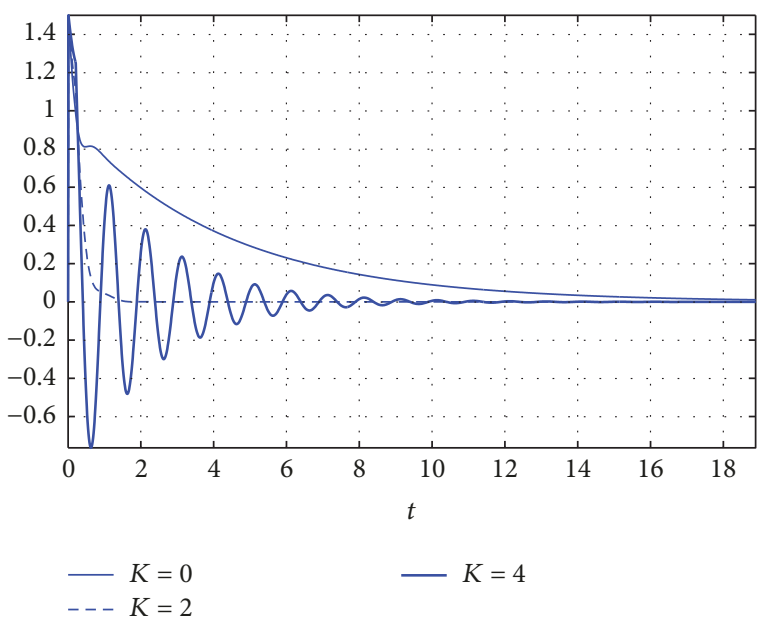

(a) $K \in\{0,2,4\}$

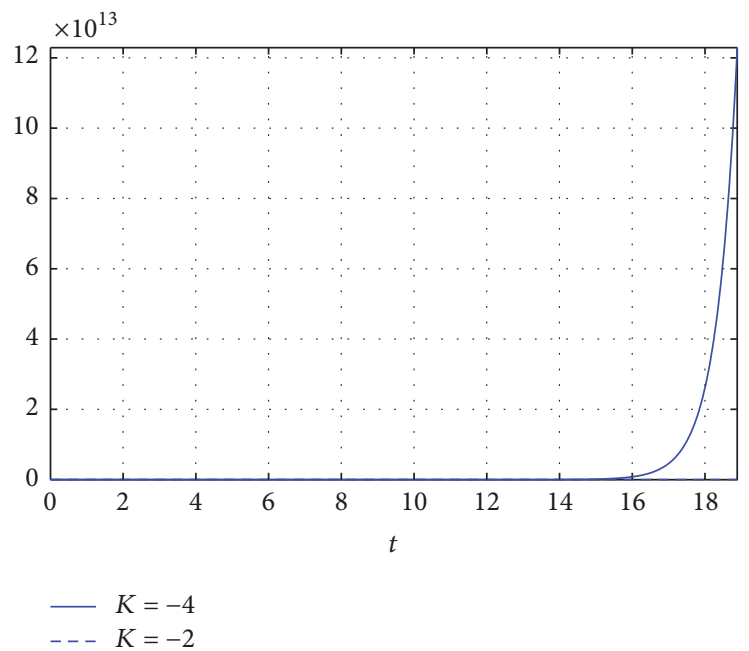

(b) $K \in\{-4,-2\}$

FIgURE 3: Time responses under the feedback gain $K \in\{-4,-2,0,2,4\}$.

Particularly, as $K \in\{-4,-2\}$ (i.e., positive feedback), the loci have one net encirclement around the origin $(0, j 0)$. By Theorem 5, the closed-loop system under $K \in\{-4,-2\}$ is unstable. In contrast, as $K \in\{0,2,4\}$ (i.e., negative feedback), the loci have no encirclement around $(0, j 0)$. By Theorem 5, the closed-loop system under $K \in\{0,2,4\}$ is asymptotically stable. In particular, the case of $K=0$ means nothing but the time-delayed system itself. Since the locus has no encirclements about the origin, the system itself is asymptotically stable.

The above stability/instability assertions are clearly reflected by the time responses of Figure 3 with the initial conditions $\phi(t)=[1,1]^{T}, \forall t \in[-0.2(=\tau), 0]$ and $u(t) \equiv$ 0 . In Figure 3(a), time responses with respect to $K \in$ $\{0,2,4\}$ converge to 0 in the steady state. In contrast, in Figure $3(\mathrm{~b})$, the time responses for $K \in\{-4,-2\}$ diverge as time grows.
Stability/instability of the closed-loop system under $K \epsilon$ $[-4,4]$ can also be examined by Corollary 6 . The simulation results are plotted in Figure 4. Clearly, the locus encirclements in terms of Corollary 6 completely coincide with those in terms of Theorem 5 .

5.1.2. Stability Analysis When $\tau$ Varies. Now we investigate stability with $\tau \in[0.1,0.5]$, while $K=4$ and $\sigma=0.3$ are fixed. The generalized loci are plotted in Figure 5 with $\tau \in\{0.1,0.2,0.3,0.4,0.5\}$, respectively. By Figure 5, when $\tau \in\{0.1,0.2\}$, the loci have zero net encirclement around the origin; thus the closed-loop time-delayed system is asymptotically stable by Theorem 5; however, when $\tau \in\{0.3,0.4,0.5\}$, each of the corresponding loci has two net encirclements around the origin; thus the closed-loop time-delayed system is unstable. In short, the longer the time delay $\tau$ in the states is, the more likely the closed-loop system tends to be unstable. 


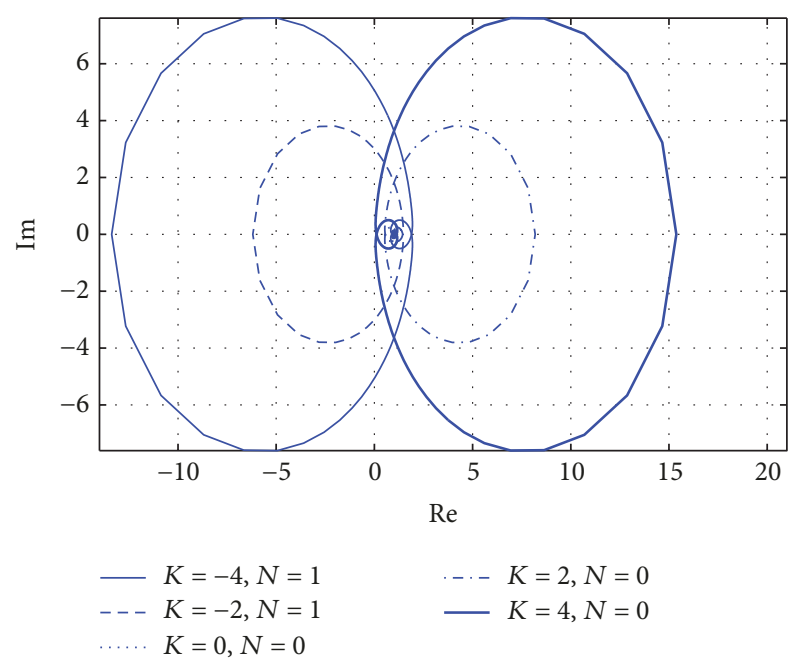

FIgURE 4: Generalized loci under the feedback gain $K \in[-4,4]$ by Corollary 6.

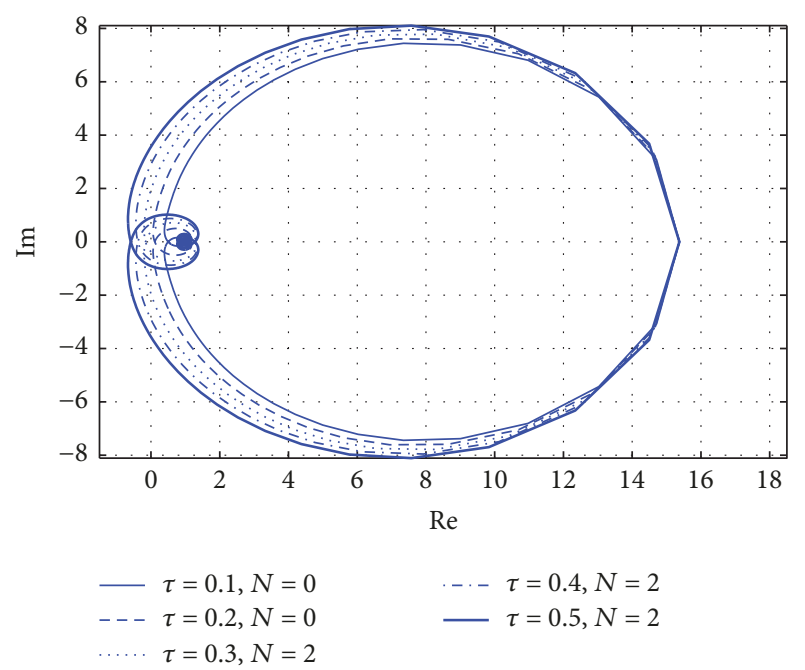

FIGURE 5: Generalized loci under $\tau \in\{0.1,0.2,0.3,0.4,0.5\}$.

5.1.3. Stability Analysis When $\sigma$ Varies. Now we investigate stability with $\sigma \in[0.2,1.0]$, while $K=4$ and $\tau=$ 0.22 are fixed. The generalized loci are plotted in Figure 6 with $\sigma \in\{0.2,0.4,0.6,0.8,1.0\}$, respectively. By Figure 6, when $\sigma \in\{0.2,1.0\}$, the loci have zero net encirclement around the origin; thus the closed-loop time-delayed system is asymptotically stable by Theorem 5; however, when $\sigma \in$ $\{0.4,0.6,0.8\}$, each of the corresponding loci has two net encirclements around the origin; thus the closed-loop timedelayed system is unstable. In short, the time delay $\sigma$ of the control input may scatter in magnitude as far as stability is concerned.

5.2. Stabilization Illustrations. Consider stabilization of the following time-delayed system by means of the static state feedback gain design procedure based on Theorem 8:

$$
\dot{x}(t)=\left[\begin{array}{cc}
0.5 & 0 \\
1 & 4
\end{array}\right] x(t)+\left[\begin{array}{cc}
0.125 & 0 \\
0.75 & -1.2
\end{array}\right] x(t-\tau)
$$

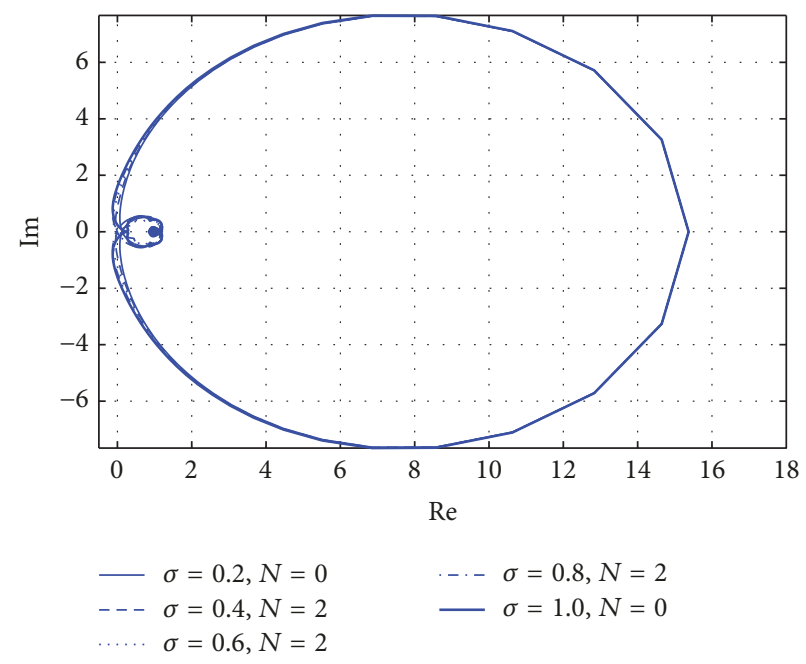

Figure 6: Generalized loci under $\sigma \in[0.2,0.4,0.6,0.8,1.0]$.

$$
+\left[\begin{array}{l}
1 \\
0
\end{array}\right] u(t)
$$

where $\tau>0$ is a single time delay in the state vector. Theorem 5 shows that the concerned system itself is unstable for any $\tau>0$. And, the pair $\left(A_{0}, B_{0}\right)$ is controllable.

Now we introduce the static state feedback $u(t)=$ $-K_{0} x(t)-K_{1} x(t-\tau)+v(t)$ with $K_{0}=\left[k_{01}, k_{02}\right]$ and $K_{1}$ $=\left[k_{11}, k_{12}\right]$. Correspondingly, the closed-loop time-delayed system is

$$
\begin{aligned}
\dot{x}(t)= & {\left[\begin{array}{cc}
0.5-k_{01} & -k_{02} \\
1 & 4
\end{array}\right] x(t) } \\
& +\left[\begin{array}{cc}
0.125-k_{11} & -k_{12} \\
0.75 & -1.2
\end{array}\right] x(t-\tau)+\left[\begin{array}{l}
1 \\
0
\end{array}\right] v(t) .
\end{aligned}
$$

Our problem here is to fix the parameters $k_{01}, k_{02}, k_{11}$, and $k_{12}$ such that the closed-loop time-delayed system is asymptotically stable.

In the closed-loop state-space equation, let us take $k_{11}=$ 0.125 and $k_{12}=0$. Then, it follows that $\left\|A_{1}-B_{0} K_{1}\right\|=1.4151$. Furthermore, the characteristic polynomial for $A_{0}-B_{0} K_{0}$ is

$$
\begin{aligned}
& \operatorname{det}\left(s I_{2}-A_{0}+B_{0} K_{0}\right) \\
& =s^{2}+\left(-4.5+k_{01}\right) s+\left(2-4 k_{01}+k_{02}\right)
\end{aligned}
$$

from which the eigenvalue formula is

$$
\begin{aligned}
& \frac{1}{2}\left[4.5-k_{01} \pm \sqrt{12.25+k_{01}^{2}+7 k_{01}-4 k_{02}}\right] \\
& =:-\alpha \pm j \rho
\end{aligned}
$$




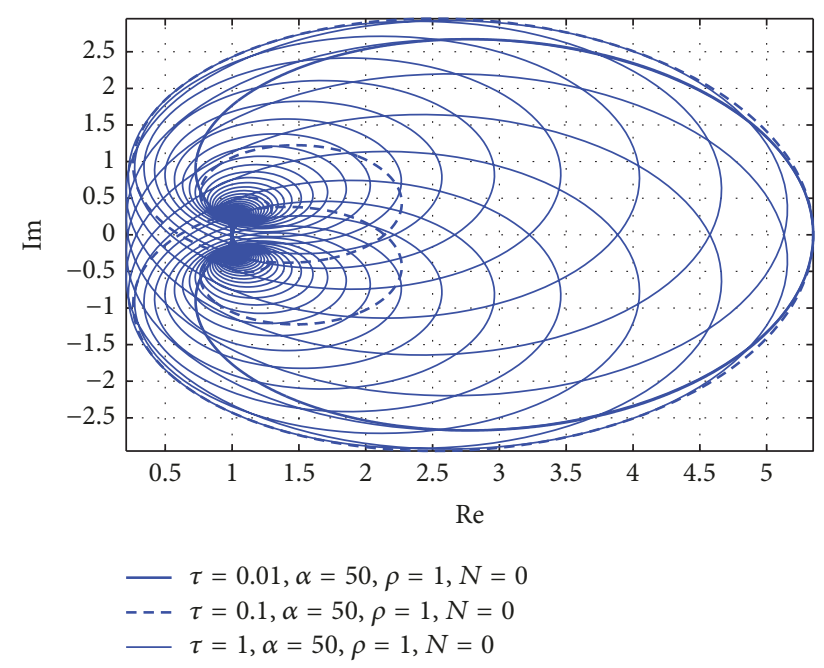

FIgURE 7: Generalized loci with respect to $\tau \in\{0.01,0.1,1\}$.

with $\alpha>0$ and $\rho \geq 0$. Based on this formula, let us take

$$
\begin{aligned}
\frac{1}{2}\left(4.5-k_{01}\right) & =-\alpha, \\
\frac{1}{2} \sqrt{12.25+k_{01}^{2}+7 k_{01}-4 k_{02}} & =j \rho,
\end{aligned}
$$

where $\alpha \geq 1.4151$ and $\rho \geq 0$. Or equivalently, we have

$$
\begin{aligned}
& k_{01}=4.5+2 \alpha, \\
& k_{02}=16+8 \alpha+\alpha^{2}+\rho^{2} .
\end{aligned}
$$

Then, the eigenvalues of $A_{0}-B_{0} K_{0}$ will be assigned at $-\alpha \pm j \rho$ as expected.

By numerical computation, when $-\alpha \pm j \rho=-50 \pm 1 j$ are assigned by means of the feedback gain $K_{0}=[104.50$, 2917.00], we can see in Figure 7 that the generalized loci for $\tau \in\{0.01,0.1,1\}$ under the same $K_{0}$ neither pass through nor encircle the origin; namely, $N=0$. Hence, the closedloop time-delayed system is stabilized under the designed $K_{0}$ and $K_{1}$ according to Theorem 5 , robustly with respect to $\tau \in[0.01,1]$.

To complete our understanding about the suggested stabilization approach, let us observe the generalized loci in Figure 8 where the eigenvalues of $A_{0}-B_{0} K_{0}$ are specified in four different cases, when $\tau=0.2$ is fixed. Clearly, in each of the last three cases the closed-loop system is stabilized, while stabilization is not attained in the first case. By the loci of Figure 8, we also see that the more left to the imaginary axis the expected eigenvalues of $A_{0}-B_{0} K_{0}$ are specified (i.e., $\left\|K_{0}\right\|$ is taken bigger), the more right to the imaginary axis the corresponding generalized loci are situated. This verifies not only the proof arguments for Proposition 7 about loci positioning, but also stability robustness with respect to $\left\|K_{0}\right\|$.

In Figure 9, time responses under the static state feedback gains $K_{0}$ adopted in Figure 8 are plotted. The convergence tendency of the time response curves coincides with the stability assertions that we conclude from Figure 8.

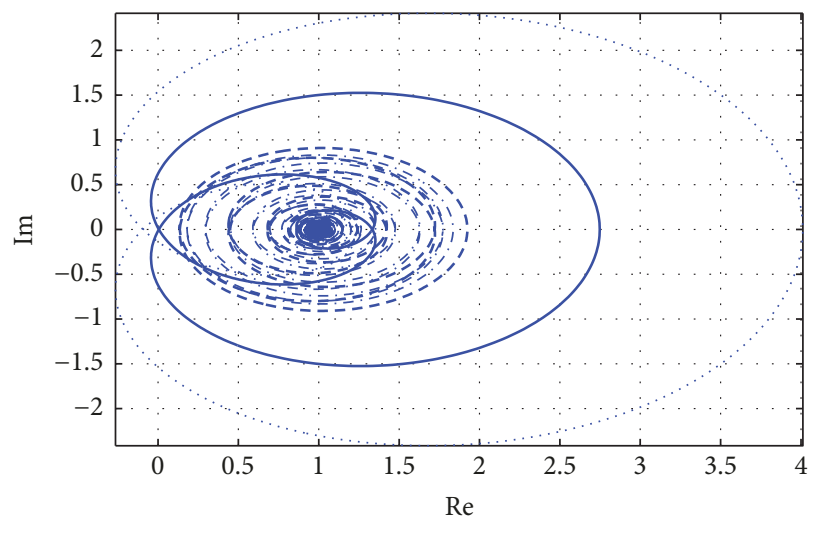

$$
\begin{aligned}
& \ldots \ldots=5, \rho=1, N=2,\left\|K_{0}\right\|=83.7721 \\
& -\alpha=10, \rho=1, N=0,\left\|K_{0}\right\|=198.5176 \\
& ---\alpha=50, \rho=1, N=0,\left\|K_{0}\right\|=2918.90 \\
& -.-\alpha=100, \rho=1, N=0,\left\|K_{0}\right\|=10819.00
\end{aligned}
$$

FIGURE 8: Generalized loci in terms of $\operatorname{det}\left(I_{n}-\left(s I_{n}-A_{0}+\right.\right.$ $\left.\left.B_{0} K_{0}\right)^{-1}\left[A_{1}-B_{0} K_{1}\right] e^{-s \tau}\right)\left.\right|_{s \in \mathcal{N}}$.

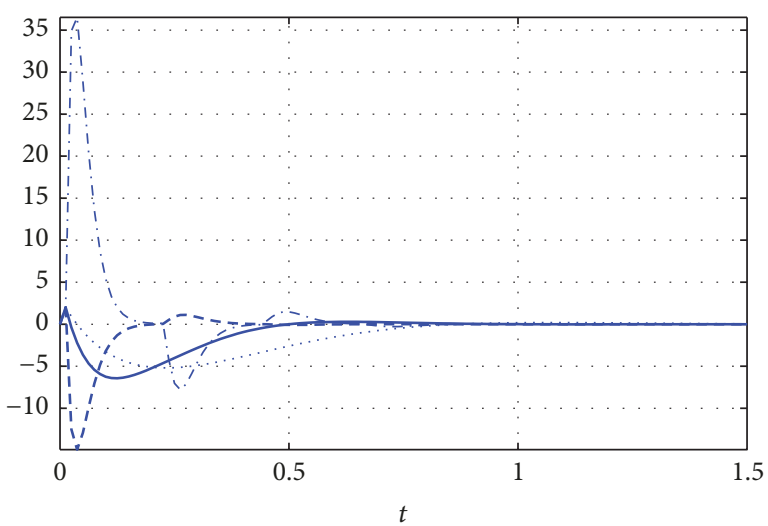

$$
\begin{aligned}
& \ldots \ldots \alpha=5, \rho=1,\left\|K_{0}\right\|=83.2721 \\
& -\alpha=10, \rho=1,\left\|K_{0}\right\|=198.5176 \\
& --\alpha=50, \rho=1,\left\|K_{0}\right\|=2918.90 \\
& -. \alpha=100, \rho=1,\left\|K_{0}\right\|=10819.00
\end{aligned}
$$

FIGURE 9: Time responses under the static state feedback gains of Figure 8.

\section{Conclusions}

In the study, we claim several complex scaling stability criteria for internal stability in linear time-delayed continuous-time systems, as summarized in Theorem 5 and its corollary. Possible advantages of the criteria include the following: (a) stability conditions are necessary and sufficient, delay-dependent, both graphically, and numerically implementable; (b) the criteria involve no open-loop characteristic roots distribution and interconnection structural facts, which are hard to know generally in time-delayed systems; (c) the locus encirclement and orientation can be self-defined after the locus is ready; (d) the criteria hold true for internal stability. Another contribution of the paper is the stabilization design in timedelayed systems, namely, Theorems 8 and 9, again based on the complex scaling technique. 
Although the results are stated only for linear timedelayed systems of retarded type, it is trivial to extend the methodology for time-delayed systems of neutral type. Due to page limitation, all details are omitted. Furthermore, how to extend the complex scaling approach to other complicated and hybrid systems, for example, linear time-varying systems and sampled-data systems with time-delayed plants, wherever some return difference relationships can be retrieved, is one of our subsequent topics. Indeed, latest results were reported in [26-28] in various types of complicated systems as reviewed in the Introduction.

\section{Conflicts of Interest}

The authors declare that they have no conflicts of interest.

\section{Acknowledgments}

The study in this paper was completed under support of the National Natural Science Foundation of China under Grant no. 61573001.

\section{References}

[1] M. Fardad and B. Bamieh, "An extension of the argument principle and Nyquist criterion to a class of systems with unbounded generators," IEEE Transactions on Automatic Control, vol. 53, no. 1, pp. 379-384, 2008.

[2] L. Pekar, R. Prokop, and R. Matusu, "A stability test for control systems with delays based on Nyquist criterion," International Journal of Mathematical Models in Applied Sciences, vol. 5, no. 7, pp. 1213-1224, 2011.

[3] J. Zhou and H. M. Qian, "Pointwise frequency responses framework for stability analysis in periodically time-varying systems," International Journal of Systems Science, vol. 48, no. 4, pp. 715-728, 2017.

[4] M. Y. Fu, A. W. Olbrot, and M. P. Polis, "Robust stability for time-delayed systems: the edge theorem and graphical tests," IEEE Transactions on Automatic Control, vol. 34, no. 8, pp. 813820, 1989.

[5] V. L. Kharitonov and A. P. Zhabko, "Robust stability of timedelay systems," IEEE Transactions on Automatic Control, vol. 39, no. 12, pp. 2388-2397, 1994.

[6] G. J. Silva, A. Datta, and S. P. Bhattacharyya, "PI stabilization of first-order systems with time delay," Automatica, vol. 37, no. 12, pp. 2025-2031, 2001.

[7] L. Vladimir and A. P. Zhabko, "Robust stability of time-delayed systems," IEEE Transactions on Automatic Control, vol. 39, no. 12, pp. 2388-2397, 1994.

[8] P. Zitek and T. Vyhlidal, "Argument-increment based stability criterion for neutral time delay systems," in Proceedings of the 16th Mediterranean Conference on Control and Automation, pp. 824-829, 2008.

[9] J. R. Partington and C. Bonnet, "Ho and BIBO stabilization of delay systems of neutral type," Systems and Control Letters, vol. 5, pp. 283-288, 2004.

[10] Q.-L. Han, "Absolute stability of time-delay systems with sectorbounded nonlinearity," Automatica, vol. 41, no. 12, pp. 21712176, 2005.
[11] J. M. Jiao and R. Zhang, "A delay decomposition approach to the stability analysis of singular systems with interval time-varying delay," Mathematical Problems in Engineering, vol. 2014, Article ID 679460, 13 pages, 2014.

[12] K. B. Shi, H. Zhu, S. M. Zhong, Y. Zeng, and Y. P. Zhang, "Improved delay-dependent robust stability criteria for a class of uncertain neutral type Lure systems with discrete and distributed delays," Mathematical Problems in Engineering, vol. 2014, Article ID 980351, 14 pages, 2014.

[13] H. Fazalinia, "Stability robustness analysis of multiple timedelayed systems using "building block" concept," IEEE Transactions on Automatic Control, vol. 52, pp. 799-810, 2007.

[14] I. Horowitz, "Some properties of delayed controls (Smith regulator)," International Journal of Control, vol. 38, no. 5, pp. 977-990, 1983.

[15] J.-H. Kim, "Delay and its time-derivative dependent robust stability of time-delayed linear systems with uncertainty," IEEE Transactions on Automatic Control, vol. 46, no. 5, pp. 789-792, 2001.

[16] N. Olgac and R. Sipahi, "A practical method for analyzing the stability of neutral type LTI-time delayed systems," Automatica, vol. 40, no. 5, pp. 847-853, 2004.

[17] N. Olgac and R. Sipahi, "An exact method for the stability analysis of time-delayed linear time-invariant (LTI) systems," IEEE Transactions on Automatic Control, vol. 47, no. 5, pp. 793797, 2002.

[18] J. D. Chen, C. H. Lien, K. K. Fan, and J. H. Chou, "Criteria for asymptotic stability of a class of neutral systems via a LMI approach," IEE Proceedings Control Theory and Applications, vol. 148, no. 6, pp. 442-447, 2001.

[19] Y. He, Q. Wang, C. Lin, and M. Wu, "Augmented Lyapunov functional and delay-dependent stability criteria for neutral systems," International Journal of Robust and Nonlinear Control, vol. 15, no. 18, pp. 923-933, 2005.

[20] J. Pan, X. Liu, and W. Xie, "Exponential stability of a class of complex-valued neural networks with time-varying delays," Neurocomputing, vol. 164, pp. 293-299, 2015.

[21] R. Rakkiyappan, G. Velmurugan, and X. Li, "Complete stability analysis of complex-valued neural networks with time delays and impulses," Neural Processing Letters, vol. 41, no. 3, pp. 435468, 2015.

[22] X. Zeng, C. Li, T. Huang, and X. He, "Stability anlaysis of complex-valued impulsive systems with time delay," Applied Mathematics and Computatio, vol. 256, pp. 75-82, 2015.

[23] J.-P. Richard, “Time-delay systems: An overview of some recent advances and open problems," Automatica, vol. 39, no. 10, pp. 1667-1694, 2003.

[24] W. Michiels and S. L. Niculescu, Stability and Stabilitzation of Time-Delayed Systems: An Eigenvalue-Based Approach, SIAM, Pennsylvania, Pa, USA, 2007.

[25] J. Zhou, H. M. Qian, and X. B. Lu, "An argument-principlebased framework for structural and spectral characteristics in linear dynamical systems," International Journal of Control, vol. 90, no. 12, pp. 2598-2604, 2017.

[26] J. Zhou and H. M. Qian, "Stability analysis of sampled-data systems via open-/closed-loop characteristic polynomials contraposition," International Journal of Systems Science, vol. 48, no. 9, pp. 1941-1953, 2017.

[27] J. Zhou, "Complex-domain stability criteria for fractional-order linear dynamical systems," IET Control Theory \& Applications, vol. 11, no. 16, pp. 2753-2760, 2017. 
[28] J. Zhou, "Interpreting Popov criteria in Lur'e systems with complex scaling stability analysis," Communications in Nonlinear Science and Numerical Simulation, vol. 59, pp. 306-318, 2018.

[29] S. Wang, Q. Jiang, and Y. Miao, "Delay-dependent stability criteria for LTI systems with multiple time delays," in Proceedings of the 2009 Chinese Control and Decision Conference, CCDC'09, pp. 5685-5690, IEEE, Guilin, China, June 2009.

[30] E. M. Stein and R. Shakarchi, Complex Analysis, Princeton University Press: Princeton and Oxford, Oxford, UK, 2003.

[31] A. W. Olbrot, "On controllability of linear systems with time delays in control," IEEE Transactions on Automatic Control, vol. 17, no. 5, pp. 664-666, 1972.

[32] K. Watanabe and M. Sato, "A process-model control for multivariable systems with multiple delays in inputs and outputs subject to unmeasurable disturbances," International Journal of Control, vol. 39, no. 1, pp. 1-17, 1984.

[33] V. B. Kolmanovskii and V. R. Nosov, "Stability of functional differential equations," in Mathematics in Science and Engineering, p. 180, Academic Press, Massachusetts, Mass, USA, 1986.

[34] D. S. Bernstein, Matrix Mathematics: Theory, Facts, and Formulas, Princeton, New Jersey, NJ, USA, 2nd edition, 2009.

[35] K. M. Zhou and J. C. Doyle, Essentials of Robust Control. Prentice Hall, Prentice Hall, New York, NY, USA, 1998.

[36] W. M. Haddad and V. Chellabonina, Nonlinear Dynamical Systems and Control: a Lyapunov-Based Approach, Princeton University Press, Oxford, UK, 2008.

[37] H. Khalil, Nonlinear Systems, Pearson Educational International Inc, New Jersey, NJ, USA, 3rd edition, 2000. 


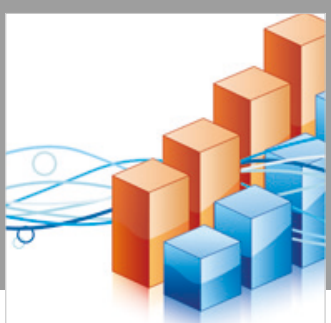

Advances in

Operations Research

\section{-n-m}
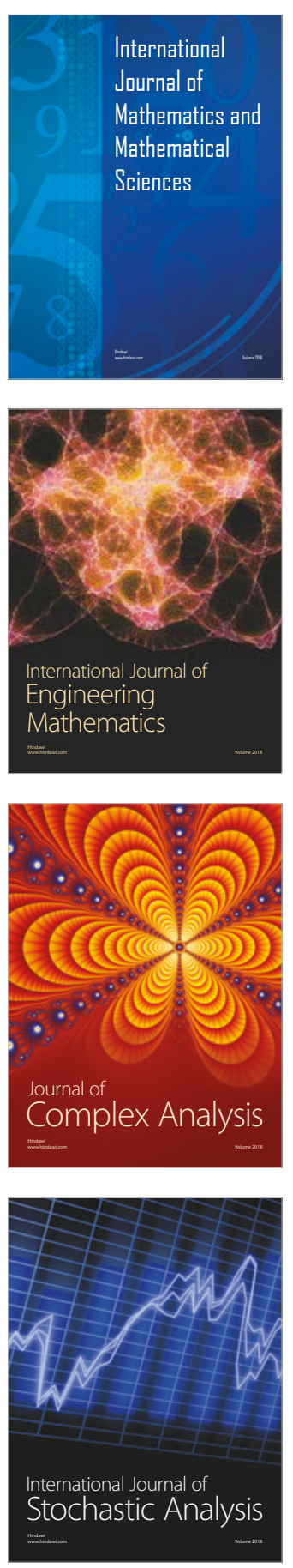
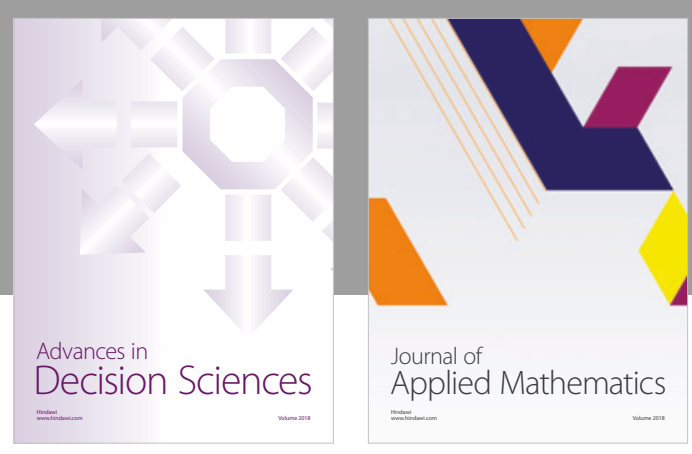

Journal of

Applied Mathematics
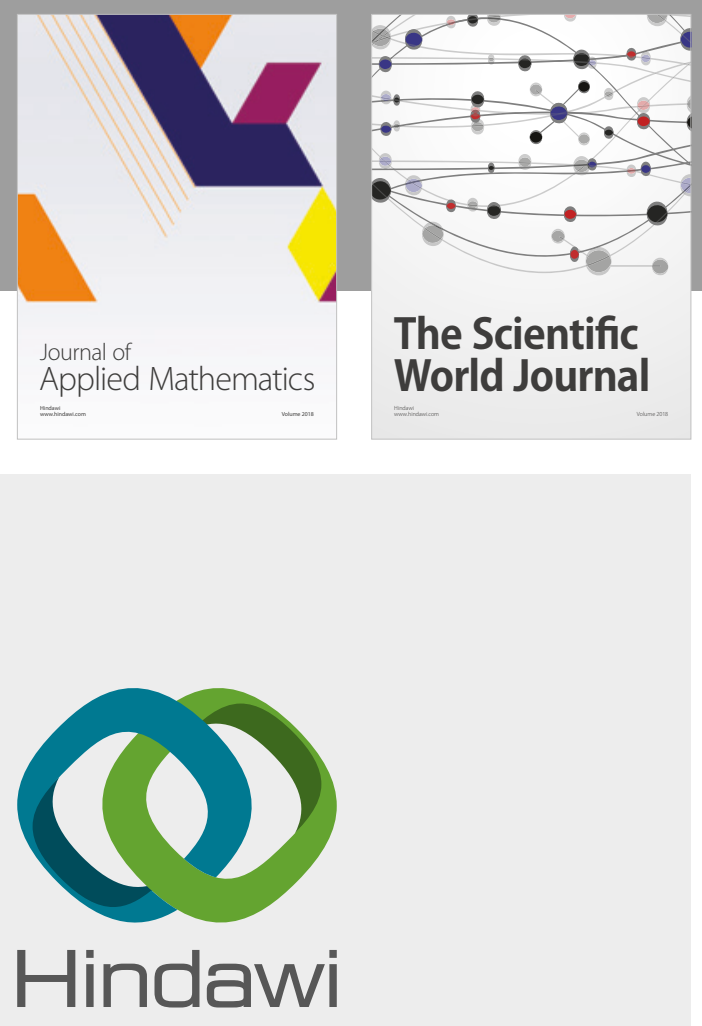

Submit your manuscripts at

www.hindawi.com

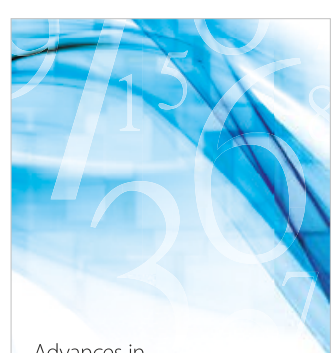

Advances in
Numerical Analysis
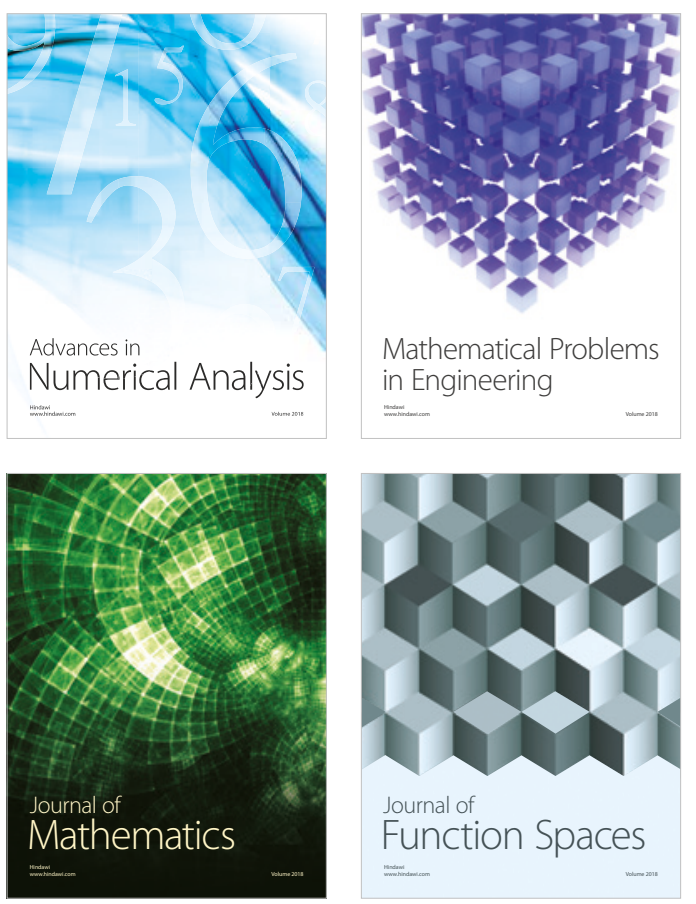

Mathematical Problems in Engineering

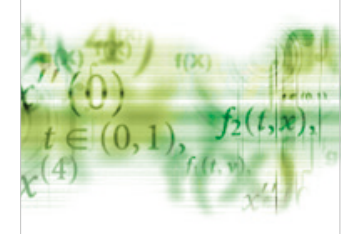

International Journal of

Differential Equations

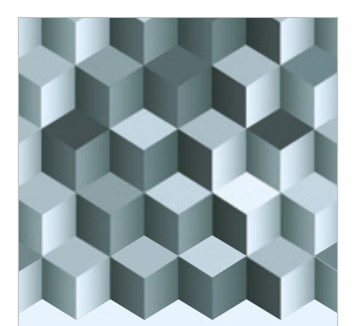

Journal of

Function Spaces

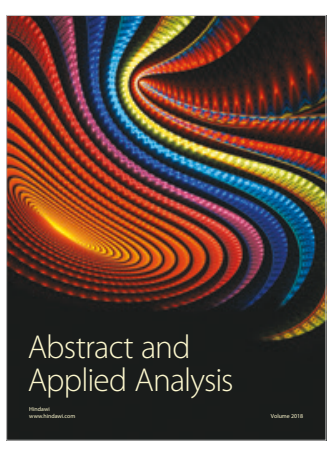

The Scientific

World Journal

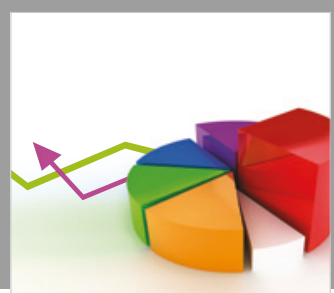

Journal of

Probability and Statistics
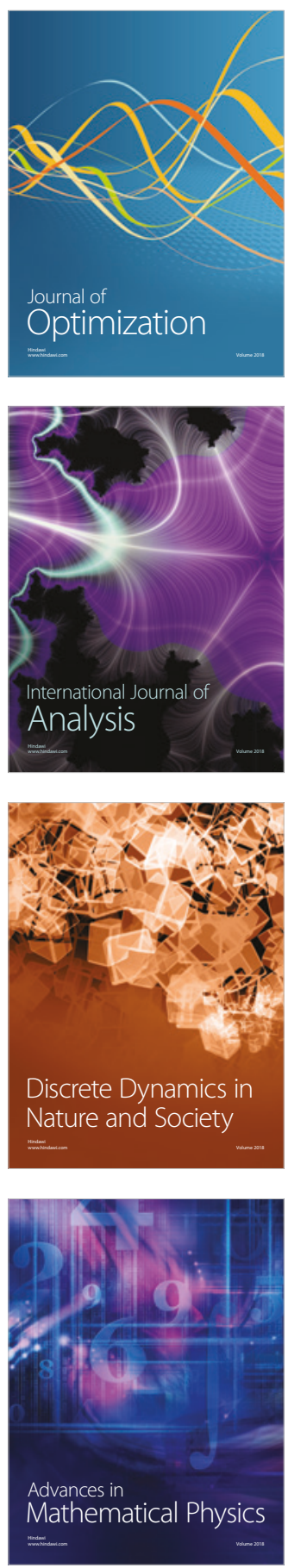